\title{
A Manufacturer-Buyers Integrated Inventory Model with Generic Distribution of Lead Times to Deliver Equal and/or Unequal Batch Sizes
}

\author{
M. A. Hoque \\ BRAC Business School, BRAC University, Dhaka-1212, Bangladesh. \\ e-mail: drmdahoque@gmail.com; mahoque332004@yahoo.com
}

Tel: +8801735905739

Arijit Bhattacharya

(Corresponding Author)

Norwich Business School, University of East Anglia, Norwich NR4 7TJ, United Kingdom. e-mail: A.Bhattacharya@uea.ac.uk; arijit.bhattacharya2005@gmail.com

Tel: +44(0)1603 597520 


\section{Highlights:}

- Propose a synchronised integrated manufacturer-buyers inventory model

- Consider generic distribution of lead times delivering equal and/or unequal batches

- Derive a minimal total cost solution technique to the model

- Consider the smallest batch size, total and unequal numbers of batches as variables

- Demonstrate significant reduction in the minimal total costs by this technique 


\title{
A Manufacturer-Buyers Integrated Inventory Model with Generic Distribution of Lead Times to Deliver Equal and/or Unequal Batch Sizes
}

\begin{abstract}
Although lead time variation is common in practice, integrated single-manufacturer multibuyer model considering this factor is unavailable in the extant literature. This article considers generic distribution of lead times of delivering equal and/or unequal batch (sub-lot) sizes of a lot in developing a synchronised integrated single-manufacturer multi-buyer model. The batch sizes are assumed to be in geometric series. The variables considered in the model are the smallest batch size, total number of batches and number of unequal batch sizes delivered from the manufacturer to buyers. The smallest batch sizes delivered to the buyers are bounded below by 1 and above by the capacity of the transport vehicle. The minimal total cost solution technique to the model is derived by the method of differentiation. Significant minimal total cost reductions by the synchronised flow is illustrated through solutions to some numerical example problems. Sensitivity analyses on increasing costs of transportation, shortage, inventory and increasing mean lead times upon the optimal solution have been performed.
\end{abstract}

Keywords: Lead time; Integrated inventory; Single-manufacturer multi-buyer; Minimal total cost; Generic distribution.

\section{Introduction}

Lead times greatly affect production and ordering decisions because of its interaction with other sources of inefficiencies in supply chains, such as the variability of consumer demand, and complexities involved in managing inventories (Ponte et al., 2018). In practice, if the vendor is geographically dispersed in an integrated vendor-buyer system, especially if it involves international transportation, the lead-time is stochastic which means that the supplier could provide the buyer with products in variable lead times (Hossain et al. 2017). This situation is particularly prevalent for festive products such as Christmas, 
Thanksgiving, New Year's Eve, Independence Day, graduation day and many such local, national and international festivities. Hossain et al. (2017) further emphasised that the joint cooperative policy ushered to some other operational or controlling issues to run such a system smoothly so that no party is at the losing end. Christensen et al. (2007) analysed surveys from 210 firms and concluded that the financial results of firms was very sensitive to lead times variability. Some researchers reported that longer the mean lead time, stronger the bullwhip effect (Chen et al., 2000; Agrawal et al., 2009; Hosoda et al., 2015). Kelepouris et al. (2008) demonstrated that an increase in mean lead times tended to result in a decreased customer satisfaction. Moreover, some researchers emphasised the effect of lead time uncertainty on supply chains management (Song et al., 2010; Kouvelis and Tang, 2011; Isotupa and Samanta, 2013; Bandaly et al., 2016; Chung et al., 2018; Ponte et al., 2018). However, collaborative solutions to integrated systems have widely shown to outperform traditional systems (e.g. Disney and Towill, 2003; Kollberg et al., 2006; Costas et al., 2015; Gunasekaran et al., 2017). Therefore, this research intends to develop a collaborative solution technique to the integrated manufacturer-buyers system considering variable lead time.

The integrated vendor-buyer(s) production-inventory literature is enriched with development of models assuming deterministic demand and lead time. Delivering lead time of a lot/batch (sub-lot) is one of the key factors in the integrated inventory models (Humair et al., 2013; Jha and Shanker, 2013; Hsiao, 2008). Researchers have devoted to the development of such models with controllable reduced lead time incurring an extra cost (e.g., Arkan and Hejazi, 2012; Yeung and Ye, 2012; Li et al., 2012; Hayya et al., 2011; Ye and Xu, 2010; Hoque, 2009; Glock, 2009; Chang et al., 2006; Hoque and Goyal, 2006; Pan and Hsiao 2005; Pan and Yang, 2002). In reality, variation in time for setting up machines, inspection, loading, transportation and unloading, etc. results in lead time deviation. Integrated inventory policies have been developed with variable lead time. Song et al. (2000) dealt with combined supply lead time and demand quantity uncertainty and reported that consideration of lead time variability performed better instead of ignoring it. Assuming stochastic demand and variable lead time, Ben-Daya and Hariga (2004) developed an integrated vendor-buyer inventory model by delivering the lot with equal batch sizes. Glock 
(2009) modified this model to permit batch shipments increasing by a fixed factor and illustrated the benefit of such shipments with the solution of a numerical example problem. Glock (2012) developed an integrated single-vendor single-buyer inventory model assuming stochastic demand and lot sizedependent variable lead time under different lead time reduction strategies. The lead time formulation of Hsiao (2008) was adopted in Glock (2012). In this model, the lead time of the first batch was formulated by considering the set up time of a machine, production and transportation times of the first batch, while the lead times for the second, third and subsequent batches till the final batch were concerned only with their transportation times. Besides, Taleizadeh et al. (2011) presented a multi-supplier multi-buyer model with lead time varying linearly and developed a harmony search solution algorithm to that model. Barron and Baron (2020) showed that variability of lead times is costly than that of perishability time, and hence recommended for this variability reduction.

Some researchers considered stochastic lead time in developing vendor-buyer integrated productioninventory models. Sajadieh et al. (2009) developed such a model with exponential distribution of lead times while allowing backordering of shortages. Sajadieh and Thorstenson (2014) considered a single/two-vendor one-buyer supply chain assuming stochastic lead time of transferring batches and minimized the overall system cost. Hoque (2013a, 2013b) presented integrated single-manufacturer single-buyer models assuming normal distribution of lead times and permitting backordering of shortages. Hoque (2013a) developed the model with the assumption of delivering a lot by a number of equal batch sizes while a number of equal and/or unequal batch sizes were used in delivering lots in Hoque (2013b). In both cases, the potential benefits of the integrated system were shown by comparative studies on solutions to numerical example problems. Thus, particular distribution of lead times in developing models for the single-manufacturer single-buyer integrated supply chain has received some attention.

Glock (2012) adopted various lead time shortening policies for transferring batches of a lot from a vendor to a buyer without considering its stochastic case. Assuming fixed and variable parts of lead time separately, Sajadieh et al. (2009) developed single-vendor single-buyer models considering exponential distribution of variable parts. However, their research did not address other practical problems under 
normal or other distributions of lead times. Also, there remains a possibility of early or late arrival of the first batch of a next lot. This situation may arise due to lack of notification to the vendor by the buyer in proper time to start processing of a next lot, generating extra inventories or shortages. In Hoque (2013a, 2013b), the reorder point is assumed to be an unknown. The time of meeting demand by this reorder point was restricted by equating it to the total time of setting up the production process and processing, inspection, loading, transfer and unloading of the first batch of a next lot. So, an extra inventory is built up by the reorder point during delivering batches (second to the last) of a same lot. As a result, the mean lead time and hence, the reorder point had not been configured appropriately in their models. Consequently, formulation of the models with such an ambiguous assumption of the mean lead time may lead to incorrect optimal solutions. In practice, the aforesaid limitations are obstacles on the way of execution of those models. Also, the literature does not report any generalised integrated singlemanufacturer multi-buyer model with stochastic lead time. In addition, synchronisation of the integrated production-distribution flow including a notification time to the manufacturer to start processing of a next lot in time is essential. Taleizadeh et al.'s (2011) model dealt with linearly variable lead time.

Driven by the above motivation, this article contributes to the extant literature by extending Hoque's (2013b) single-manufacturer single-buyer model to a generalised single-manufacturer multi-buyer case by updating these assumptions. The production flow of this updated model is synchronised by transferring a lot with equal and/or unequal batch sizes and creating a notification point to the manufacturer to start production of a next lot for transporting the first batch of that lot to the buyers in time. In this extension, we consider an improved independent stochastic mean lead time. The general model has been embedded with generic distribution of this mean lead time to facilitate wider application. The variables considered in the model are the smallest batch size, total number of batches and number of unequal batch sizes transferred from the manufacturer to buyers. The smallest batch size is constrained by the capacity of the transport vehicle and the smallest positive integer. A minimal total cost solution technique to the model by the method of differentiation is derived, and its potential significance is highlighted with illustration of solutions to numerical example problems. The numerical examples resemble some cases of an exhaust 
silencer manufacturer selling their products to car producing plants with specific annual rate of demands per year. Sensitivity analyses on the effects of increasing parameter values of transportation, shortage, inventory costs and increasing mean lead time, upon the minimal total cost solutions are carried out. Finally, the shortcomings of the current model are highlighted with a proposal to conduct further studies on the topic.

This paper is organised as follows. Section 2 presents the problem along with the proposed model. Section 3 explores solution algorithm details to achieve the minimal total cost solution to the model. Next, in section 4, numerical example problems are illustrated to elucidate the efficacy of the solution technique. This section further provides detailed analyses on the potential benefits of the proposed model through comparative studies on the results of single-manufacturer single-buyer numerical problems, followed by sensitivity analyses. Section 5 concludes by highlighting the paper findings, limitations and future research scope.

\section{Model Formulation}

\subsection{Assumptions and notation}

The assumptions made in developing the model are as follows:

i) Production and demand rates over an infinite time horizon are deterministic constant, where the former is greater than the latter;

ii) The manufacturer processes a lot of a product and transfers it to the buyers with a number of equal-and/or unequal- sized batches;

iii) The buyers start to meet demands at the same time;

iv) Planned shortages are allowed and backordered completely;

v) Shortage cost/year is higher than holding cost/year at a buyer (Fujiwara and Sedarage, 1997);

vi) Lead times to replenish buyers' orders follow a continuous probability distribution function and lead time of a succeeding batch is independent of the lead time preceding it; 
vii) Buyers' inventories are reviewed continuously, and the next batch is ordered when the current inventory hits a planned reorder point;

viii) Sharing of benefit of the manufacturer-buyers integrated inventory system through negotiation is agreed with a provision of extra financial incentives to the potential buyers;

ix) The orders crossing in time is not allowed (Sajadieh et al., 2009).

Following notation are used in developing the model:

\begin{tabular}{cl}
\hline Notations & \multicolumn{1}{c}{ Description } \\
\hline$D$ & Rate of demand per year for all buyers \\
\hline$P$ & Rate of production per year assuming $P>D,(k=P / D)$ \\
\hline $\mathrm{Q}$ & Size of a lot delivered from the manufacturer to the buyers \\
\hline$z$ & The smallest of the batches of $Q$ \\
\hline$n$ & Total number of batches in a lot $Q$ \\
\hline$b$ & Number of unequal batch sizes $(m \leq n)$ \\
\hline$S$ & Number of buyers \\
\hline$S_{t}$ & Manufacturer's set up cost/set up \\
\hline$h$ & Manufacturer's set up time/set up \\
\hline$g$ & Capacity of the transport vehicle \\
\hline For the $i$ th buyer ( $\forall i=1,2, \ldots, b)$ \\
\hline$D_{i}$ & Annual rate of demand $\left(\mathrm{D}=\sum_{\mathrm{i}=1}^{\mathrm{b}} \mathrm{D}_{\mathrm{i}}\right)$ \\
\hline$Q_{i}$ & Lot size \\
\hline$z_{i}$ & The smallest batch size of the lot $Q_{i}$ \\
\hline$h_{i}$ & Inventory carrying cost per item per year $\left(h_{i}>h\right)$ \\
\hline$\pi_{\mathrm{i}}$ & Shortage cost/unit/year $\left(h_{i}<\pi_{i}\right)$ \\
\hline$S_{i}$ & Cost of placing an order \\
\hline$T_{i}$ & Transportation cost for transporting a batch from the manufacturer \\
\hline$l_{i}$ & Lead time to replenish buyer's order \\
\hline$\sigma_{\mathrm{i}}$ & Standard deviation of the lead times \\
\hline$t_{i}$ & Usual inspection, loading, transfer and unloading time $(\mathrm{yr})$ \\
\hline $\mathrm{t}_{\mathrm{i}}^{\prime}$ & Return time ( yr) of the transport vehicle \\
\hline $\mathrm{r}_{\mathrm{i}}$ & Buyer's notification point \\
\hline
\end{tabular}




\subsection{The Model}

\subsubsection{The total cost function}

We assume that a manufacturer produces a homogenous product and delivers it to multiple buyers. Our aim is to minimise the expected annual total cost of ordering batches, set up of the production process, inventory holding during production and meeting demands, shortage and transportation of batches, subject to some constraints provided later. Let a manufacturer processes and transfers a lot of size $Q$ to $b$ buyers by $m$ batches of size $z, k z, k^{2} z, \ldots k^{m-1} z$ and $n-m$ batches of size $k^{m-1} z$. A part of each batch $k^{j-1} z(j=1,2 \ldots m)$ is transferred to the buyer $i(i=1,2, \ldots, b)$ by batches of size $k^{j-1} z_{i}$, where $k^{j-1} z_{i}=D_{i}\left(k^{j-1} z\right) / D$ or $k^{j-1} z_{i} / D_{i}=k^{j-1} z / D$ as in Hoque (2008), so that $k^{j-1} z=k^{j-1} \sum_{i=1}^{b} z_{i}$.

Thus $Q_{i}=z_{i}+k z_{i}+\ldots+k^{m-1} z_{i}+(n-m) k^{m-1} z_{i}$ and $Q=\sum_{=1}^{b} Q_{i}=\sum_{i=1}^{b}\left(z_{i}+k z_{i}+\ldots+k^{m-1} z_{i}+(n-m) k^{m-1} z\right)$

$$
\begin{array}{r}
=z+k z+k^{2} z+\ldots+k^{m-1} z+(n-m) k^{m-1} z=z\left(\sum_{j=1}^{m} k^{j-1}+(n-m) k^{m-1}\right) \\
z=\frac{Q}{\sum_{j=1}^{m} k^{j-1}+(n-m) k^{m-1}} \text { and } z_{i}=\frac{Q_{i}}{\sum_{j=1}^{m} k^{j-1}+(n-m) k^{m-1}}
\end{array}
$$

Also, $Q_{i}=z_{i}+k z_{i}+k^{2} z_{i}+\ldots+k^{m-1} z_{i}+(n-m) k^{m-1} z_{i}$

$=D_{i} z / D+D_{i}(k z) / D+D_{i}\left(k^{2} z\right) / D+\ldots+D_{i}\left(k^{m-1} z\right) / D+(n-m) D_{i}\left(k^{m-1} z\right) / D=D_{i} Q / D \Rightarrow Q / D=Q_{i} / D_{i}$.

In addition, $D_{i}\left(k^{j-1} z\right) / D=k^{j-1} z_{i} \Rightarrow(P / D)\left\{D_{i}\left(k^{j-1} z\right) / P\right\}=k^{j-1} z_{i}$

$\Rightarrow k\left(k^{j-1} z\right) / P=k^{j-1} z_{i} / D_{i} \Rightarrow k^{j} z / P=k^{j-1} z_{i} / D_{i}=k^{j-1} z / D\left[\because z_{i}=D_{i}(z) / D\right]$

Accordingly, the production time of any unequal- sized batch equals the time of meeting demand by the previous batch, and thus synchronizes the production flow of supplying a product to multiple buyers with equal and/or unequal batch sizes after its production at the manufacturer. 
Since $P>D$, the next batch of a lot can always be made available at the manufacturer when the current one finishes at the buyers $\left(\because k^{j} z / P=k^{j-1} z_{i} / D_{i}=k^{j-1} z / D\right)$. Since $t_{i}$ is the usual time for inspection, loading, transportation and unloading of a batch for buyer $i$, it is assumed that a buyer orders for a next batch when his/her stock level hits the reorder point $D_{i} t_{i}$. The next batch is supposed to arrive at the buyer $i$ when these units finish there. So, the mean lead time is $t_{i}$. If the next batch is supplied from the same lot, it is always available at the manufacturer and hence maintains its mean lead time $t_{i}$. However, if the next batch is the first batch of a next lot, the manufacturer needs the set up time $S_{t}$, processing time of the first batch $z / P$, and the time $t_{i}$. So, the manufacturer must be notified by the buyer $i, S_{t}+z / P+t_{i}$ time before finishing the current batch at this buyer, thus maintaining the same mean lead time. Let us define the time $S_{t}+z / P+t_{i}$ as the notification time. Thus, a buyer $i$ preserves a notification point.

Initially, a batch $z$ is processed at the manufacturer and then the batch $z_{i}=D_{i} z / D$ is delivered to buyer $i$ in time $t_{i .}$. The batch $z_{i}$ meets demand in time $z_{i} / D_{i}$, generating an inventory $z_{i}^{2} /\left(2 D_{i}\right)$. If $t_{m}=\max _{i}\left(t_{i}\right)$, each batch $k^{j-1} z_{i}$ is kept at the manufacturer for time $t_{m}-t_{i}$, and then it is supposed to deliver to buyer $i$ in time $t_{i}$ so that all buyers start to meet demands at the same time. Thus each batch $k^{j-1} z_{i}$ is supposed to remain idle for time $t_{m}-t_{i}$ (at the manufacturer) $+t_{i}$ (during transportation) $=t_{m}$. The sum of the inventories at the buyers created by the batch $k^{j-1} z$ is given by:

$=\frac{k^{2(j-1)} z_{1}^{2}}{2 D_{1}}+\frac{k^{2(j-1)} z_{2}^{2}}{2 D_{2}}+\ldots+\frac{k^{2(j-1)} z_{b}^{2}}{2 D_{b}}=\frac{k^{2(j-1)}}{2}\left(z_{1}+z_{2}+\ldots+z_{b}\right) \frac{z}{D}=\frac{k^{2(j-1)} z^{2}}{2 D} \quad\left[\because \frac{z_{i}}{D_{i}}=\frac{z}{D}\right]$

The buyer's inventory per year is given by:

$$
=\frac{D_{i}}{Q_{i}}\left\{\sum_{j=1}^{m} \frac{k^{2(j-1)} z^{2}}{2 D}+(n-m) \frac{k^{2(m-1)} z^{2}}{2 D}\right\}=\frac{z^{2}}{2 Q}\left\{\sum_{j=1}^{m} k^{2(j-1)}+(n-m) k^{m-1}\right\}\left[\because \frac{D}{Q}=\frac{D_{i}}{Q_{i}}\right] .
$$

The manufacturer-buyers joint inventory pattern of transferring a lot by 4 batches is depicted in Figure 1, where the last two are of equal-sized. 


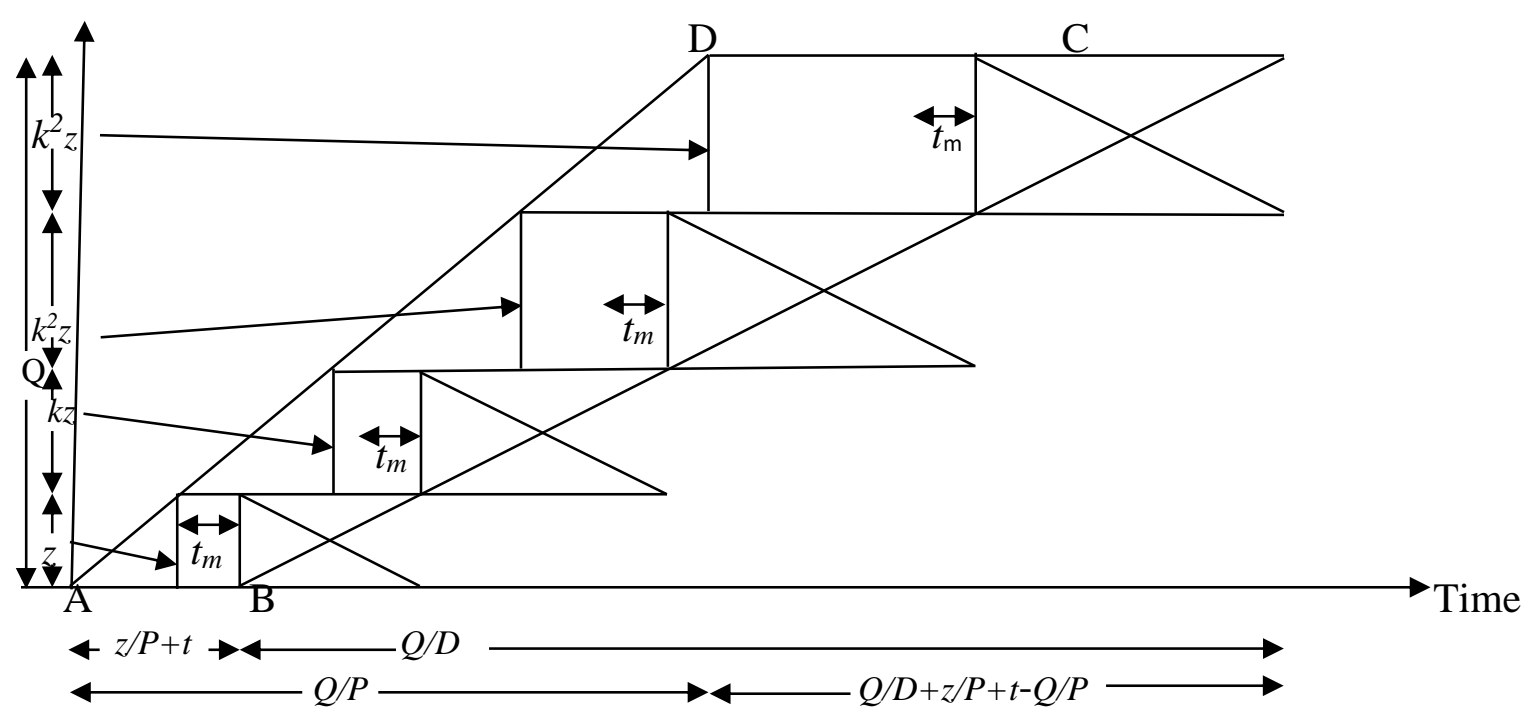

Figure 1: The joint inventory of the manufacturer and the buyers when $n=4, m=3$

Area of the trapezium $\mathrm{ABCD}$ denotes manufacturer-buyers joint inventory per cycle, and hence their joint inventory per year is given by $\frac{z}{k}+D t_{m}+\frac{Q}{2}\left(1-\frac{1}{k}\right)$.

Since manufacturer's inventory is the joint inventory minus the buyers' inventory, the inventory cost of the manufacturer per year is given by:

$$
\left[\frac{z}{k}+D t_{m}+\frac{Q}{2}\left(1-\frac{1}{k}\right)-\frac{z\left\{\sum_{j=1}^{m} k^{2(j-1)}+(n-m) k^{2(m-1)}\right\}}{2\left\{\sum_{j=1}^{m} k^{j-1}+(n-m) k^{m-1}\right\}}\right] h
$$

Because of variations in the lead time $l_{i}$, a batch may arrive early or late, leading to deviation from the mean lead time $t_{i}$. We assume that this variation in the lead time follows a continuous probability distribution function $f_{L_{i}}\left(l_{i}\right)$. By embedding this function we calculate various concerned costs of the considered problem following Hoque (2013b) which are shown in the sub-sections below.

\section{(i) Inventory cost when a batch arrives early or on time}

Here the inventory created during meeting demand from the reorder point and from the time of arrival of a batch until it reaches to the next reorder point of a cycle are calculated separately. Their sum is 
multiplied by the number of cycles in a year in obtaining the inventory per year. After simplification the expected inventory cost per year for all the buyers is given by:

$$
\sum_{i=1}^{b}\left[D_{i} h_{i} \int_{0}^{t_{i}}\left\{\frac{z\left(\sum_{j=1}^{m} k^{2(j-1)}+(n-m) k^{2(m-1)}\right)}{2 D\left(\sum_{j=1}^{m} k^{j-1}+(n-m) k^{m-1}\right)}+\left(t_{i}-l_{i}\right)\right\}\right] f_{L_{i}}\left(l_{i}\right) d l_{i}
$$

\section{(ii) Shortage and the inventory costs when a batch arrives late}

When a batch $k^{j-1} z_{i}$ arrives late to the buyer $i$ limiting lead time $l_{i}$ in the range $t_{i}<l_{i}<t_{i}+k^{j-1} z_{i} / D_{i}$, the part of the shortage cost at the buyer $i$ per year is obtained as:

$$
\frac{D D_{i} \pi_{i}}{2 z\left\{\sum_{j=1}^{m} k^{j-1}+(n-m) k^{m-1}\right\}}\left[\sum_{j=1}^{m} \int_{t_{i}}^{t_{i}+k^{j-1} z / D}\left(l_{i}-t_{i}\right)^{2} f_{L_{i}}\left(l_{i}\right) d l_{i}+(n-m) \int_{t_{i}}^{t_{i}+k^{m-1} z / D}\left(l_{i}-t_{i}\right)^{2} f_{L_{i}}\left(l_{i}\right) d l_{i}\right] .
$$

Further, the part of the inventory cost at this buyer per year in this case is derived as:

$$
\begin{aligned}
\frac{D D_{i} h_{i}}{2 z\left(\sum_{j=1}^{m} k^{j-1}+(n-m) k^{m-1}\right)}\left[\sum_{j=1}^{m} \int_{t_{i}}^{t_{i}+k^{j-1} z / D}\left(k^{j-1} \frac{z}{D}+t_{i}-l_{i}\right)^{2} f_{L_{i}}\left(l_{i}\right) d l_{i}\right. \\
\left.+(n-m) \int_{t_{i}}^{t_{i}+k^{m-1} z / D}\left(k^{j-1} \frac{z}{D}+t_{i}-l_{i}\right)^{2} f_{L_{i}}\left(l_{i}\right) d l_{i}\right]
\end{aligned}
$$

So, the total inventory plus shortage costs per year for all buyers in this case is given by expression (4):

$$
\begin{aligned}
& \frac{D}{2 z\left\{\sum_{j=1}^{m} k^{j-1}+(n-m) k^{m-1}\right\}} \sum_{i=1}^{b} D_{i} {\left[\sum_{j=1}^{m}\left\{\int_{t_{i}}^{t_{i}+k^{j-1} z / D}\left(\pi_{i}\left(l_{i}-t_{i}\right)^{2}+h_{i}\left(k^{j-1} \frac{z}{D}+t_{i}-l_{i}\right)^{2}\right)\right\} f_{L_{i}}\left(l_{i}\right) d l_{i}\right.} \\
&\left.+(n-m)\left\{\int_{t_{i}}^{t_{i}+k^{m-1} z / D}\left(\pi_{i}\left(l_{i}-t_{i}\right)^{2}+h_{i}\left(k^{j-1} \frac{z}{D}+t_{i}-l_{i}\right)^{2}\right) f_{L_{i}}\left(l_{i}\right) d l_{i}\right\}\right] \ldots
\end{aligned}
$$

When a batch $k^{j-1} z_{i}$ arrives late to the buyer $i$ by limiting lead time $l_{i}$ in the range $t_{i}+k^{j-1} z_{i} / D_{i}<l_{i}<\infty$, the shortage cost per year for all the buyers is given by expression (5):

$\frac{1}{\sum_{j=1}^{m} k^{j-1}+(n-m) k^{m-1}} \sum_{i=1}^{b} D_{i} \pi_{i}\left[\sum_{j=1}^{m} \int_{t_{i}+k^{j-1} z / D}^{\infty} k^{j-1}\left\{l_{i}-t_{i}-\frac{k^{j-1} z}{2 D}\right\} f_{L i}\left(l_{i}\right) d l_{i}\right.$ 


$$
\left.+(n-m) \int_{t_{i}+k^{m-1} z / D}^{\infty} k^{m-1}\left\{l_{i}-t_{i}-\frac{k^{m-1} z}{2 D}\right\} f_{L i}\left(l_{i}\right) d l_{i}\right]
$$

If a batch $k^{j-1} z_{i}$ arrives late by the time $l_{i}-t_{i}$, limiting $l_{\mathrm{i}}$ in the range $t_{i}<l_{i} \leq t_{i}+k^{j-1} z_{i} / D_{i}$, the extra inventory cost per year (assuming that it is kept at the manufacturer for this time) in this case is given by:

$$
\frac{D_{i} h}{\sum_{j=1}^{m} k^{j-1}+(n-m) k^{m-1}}\left[\sum_{j=1}^{m}\left\{k^{j-1} \int_{t_{i}}^{t_{i}+k^{j-1} z / D}\left(l_{i}-t_{i}\right) f_{L_{i}}\left(l_{i}\right) d l_{i}\right\}+(n-m)\left\{k^{m-1} \int_{t_{i}}^{t_{i}+k^{m-1} z / D}\left(l_{i}-t_{i}\right) f_{L_{i}}\left(l_{i}\right) d l_{i}\right\}\right]
$$

If a batch $k^{j-1} z_{i}$ arrives late by a time $l_{i}-t_{i}$, limiting $l_{i}$ in the range $t_{i}+k^{j-1} z_{i} / D_{i}<l_{i}<\infty$, then the contribution to the inventory cost of the manufacturer per year by this extra inventory is given by: $\frac{D_{i} h}{\sum_{j=1}^{m} k^{j-1}+(n-m) k^{m-1}}\left[\sum_{j=1}^{m}\left\{k^{j-1} \int_{t_{i}+k^{j-1} z / D}^{\infty}\left(l_{i}-t_{i}\right) f_{L_{i}}\left(l_{i}\right) d l_{i}\right\}+(n-m)\left\{k^{m-1} \int_{t_{i}+k^{m-1} z / D}^{\infty}\left(l_{i}-t_{i}\right) f_{L_{i}}\left(l_{i}\right) d l_{i}\right\}\right]$.

Adding this expression to the previous one, we find the total extra inventory cost per year at the manufacturer as $D_{i} h \int_{t_{i}}^{\infty}\left(l_{i}-t_{i}\right) f_{L_{i}}\left(l_{i}\right) d l_{i}$. If a batch $k^{j-1} z_{i}$ arrives early to the buyer $i$ by $\left(t_{i}-l_{i}\right)$, limiting $l_{i}$ in the range $0<l_{i} \leq t_{i}$, then the inventory cost per year is obtained as:

$$
\frac{D_{i} z_{i} h}{Q_{i}}\left\{\sum_{j=1}^{m} k^{j-1}+(n-m) k^{m-1}\right\} \int_{0}^{t_{i}}\left(t_{i}-l_{i}\right) f_{L_{i}}\left(l_{i}\right) d l_{i}=D_{i} h \int_{0}^{t_{i}}\left(t_{i}-l_{i}\right) f_{L_{i}}\left(l_{i}\right) d l_{i}
$$

Thus, the net extra inventory cost for all buyers per year is given by expression (6):

$$
h \sum_{i=1}^{b} D_{i}\left\{\int_{t_{i}}^{\infty}\left(l_{i}-t_{i}\right) f_{L_{i}}\left(l_{i}\right) d l_{i}-\int_{0}^{t_{i}}\left(t_{i}-l_{i}\right) f_{L_{i}}\left(l_{i}\right) d l_{i}\right\}=h \sum_{i=1}^{b} D_{i} \int_{0}^{\infty}\left(l_{i}-t_{i}\right) f_{L_{i}}\left(l_{i}\right) d l_{i}
$$

\section{(iii) Sum of set up, ordering and transportation cost}

Total cost of set up of the production process, ordering and transportation of batches per year is given by expression (7):

$$
\frac{D\left[S+\sum_{i=1}^{b}\left(S_{i}+n T_{i}\right)\right]}{Q}=\frac{D\left[S+\sum_{i=1}^{b}\left(S_{i}+n T_{i}\right)\right]}{z\left\{\sum_{j=1}^{m} k^{j-1}+(n-m) k^{m-1}\right\}}
$$




\section{(iv) The total cost function}

Adding the derived costs of expressions (2), (3), (4), (5), (6) and (7), and then substituting for $Q=z\left(\sum_{j=1}^{m} k^{j-1}+(n-m) k^{m-1}\right)$ from (1), the total cost function, $T C(z, n, m)$, is found as follows:

$$
\begin{aligned}
& \sum_{i=1}^{b}\left[D_{i} h_{i} \int_{0}^{t_{i}}\left\{\frac{z\left\{\sum_{j=1}^{m} k^{2(j-1)}+(n-m) k^{2(m-1)}\right\}}{2 D\left\{\sum_{j=1}^{m} k^{j-1}+(n-m) k^{m-1}\right\}}+\left(t_{i}-l_{i}\right)\right\}\right] f_{L_{i}}\left(l_{i}\right) d l_{i} \\
& +\frac{1}{\left\{\sum_{j=1}^{m} k^{j-1}+(n-m) k^{m-1}\right\}} \sum_{i=1}^{b} D_{i}\left[\sum_{j=1}^{m}\left\{\int_{t_{i}}^{t_{i}+k^{j-1} z / D} \frac{D}{2 z}\left(\pi_{i}+h_{i}\right)\left(l_{i}-t_{i}\right)^{2}-h_{i}\left\{k^{j-1}\left(l_{i}-t_{i}\right)-\frac{z k^{2(j-1)}}{2 D}\right\}\right\} f_{L_{i}}\left(l_{i}\right) d l_{i}\right. \\
& \left.+(n-m)\left\{\int_{t_{i}}^{t_{i}+k^{m-1} z / D} \frac{D}{2 z}\left(\pi_{i}+h_{i}\right)\left(l_{i}-t_{i}\right)^{2}-h_{i}\left\{k^{m-1}\left(l_{i}-t_{i}\right)-\frac{z k^{2(m-1)}}{2 D}\right\} f_{L_{i}}\left(l_{i}\right) d l_{i}\right\}\right] \\
& +\frac{1}{\sum_{j=1}^{m} k^{j-1}+(n-m) k^{m-1} \sum_{i=1}^{b} D_{i} \pi_{i}\left[\sum_{j=1}^{m} \int_{t_{i}+k^{j-1} z / D}^{\infty} k^{j-1}\left\{l_{i}-t_{i}-\frac{k^{j-1} z}{2 D}\right\} f_{L i}\left(l_{i}\right) d l_{i}\right.} \\
& \left.+(n-m) \int_{t_{i}+k^{m-1} z / D}^{\infty} k^{m-1}\left\{l_{i}-t_{i}-\frac{k^{m-1} z}{2 D}\right\} f_{L i}\left(l_{i}\right) d l_{i}\right]+h \sum_{i=1}^{b} D_{i} \int_{0}^{\infty}\left(l_{i}-t_{i}\right) f_{L_{i}}\left(l_{i}\right) d l_{i}+\frac{D}{z\left\{\sum_{j=1}^{m} k^{j-1}+(n-m) k^{m-1}\right\}} \\
& +\left[\frac{z}{k}+D t_{m}+\frac{z\left(\sum_{j=1}^{n} k^{j-1}+(n-m) k^{m-1}\right)}{2}\left(1-\frac{1}{k}\right)-\frac{z\left\{\sum_{j=1}^{m} k^{2(j-1)}+(n-m) k^{2(m-1)}\right\}}{2\left\{\sum_{j=1}^{m} k^{j-1}+(n-m) k^{m-1}\right\}}\right] h
\end{aligned}
$$

Applying the equation:

$$
\int_{t_{i}}^{t_{i}+k^{j-1} z / D}\left\{\left(l_{i}-t_{i}-\frac{k^{j-1} z}{2 D}\right)\right\} f_{L_{i}}\left(l_{i}\right) d l_{i}=\int_{t_{i}}^{\infty}\left\{\left(l_{i}-t_{i}-\frac{k^{j-1} z}{2 D}\right)\right\} f_{L_{i}}\left(l_{i}\right) d l_{i}-\int_{t_{i}+k^{j-1} z / D}^{\infty}\left\{\left(l_{i}-t_{i}-\frac{k^{j-1} z}{2 D}\right)\right\} f_{L_{i}}\left(l_{i}\right) d l_{i}, \quad \text { it }
$$

transforms to expression (8):

$$
\begin{aligned}
& T C(z, n, m)=\sum_{i=1}^{b}\left[D_{i} h_{i} \int_{0}^{t_{i}}\left\{\frac{z\left\{\sum_{j=1}^{m} k^{2(j-1)}+(n-m) k^{2(m-1)}\right\}}{2 D\left\{\sum_{j=1}^{m} k^{j-1}+(n-m) k^{m-1}\right\}}+\left(t_{i}-l_{i}\right)\right\} f_{L_{i}}\left(l_{i}\right) d l_{i}\right. \\
& +\frac{D}{2 z\left\{\sum_{j=1}^{m} k^{j-1}+(n-m) k^{m-1}\right\}} \sum_{i=1}^{b} D_{i}\left(\pi_{i}+h_{i}\right)\left[\sum_{j=1}^{m} \int_{t_{i}}^{t_{i}+k^{j-1} z / D}\left(\left(l_{i}-t_{i}\right)-\frac{z k^{j-1}}{D}\right)^{2} f_{L_{i}}\left(l_{i}\right) d l_{i}\right.
\end{aligned}
$$




$$
\begin{gathered}
\left.+\frac{D\left[S+\sum_{i=1}^{b}\left(S_{i}+n T_{i}\right)\right]}{z\left\{\sum_{j=1}^{m} k^{j-1}+(n-m) k^{m-1}\right\}}+(n-m)\left\{\int_{t_{i}}^{t_{i}+k^{m-1} z / D}\left(\left(l_{i}-t_{i}\right)-\frac{z k^{m-1}}{D}\right)^{2} f_{L_{i}}\left(l_{i}\right) d l_{i}\right\}\right] \\
+\frac{1}{\sum_{j=1}^{m} k^{j-1}+(n-m) k^{m-1}} \sum_{i=1}^{b} D_{i} \pi_{i}\left[\sum_{j=1}^{m} k^{j-1} \int_{t_{i}}^{\infty}\left\{\left(l_{i}-t_{i}-\frac{k^{j-1} z}{2 D}\right)\right\} f_{L_{i}}\left(l_{i}\right) d l_{i}\right. \\
\left.+(n-m) k^{m-1} \int_{t_{i}}^{\infty}\left\{\left(l_{i}-t_{i}-\frac{k^{m-1} z}{2 D}\right)\right\} f_{L_{i}}\left(l_{i}\right) d l_{i}\right]+h \sum_{i=1}^{b} D_{i} \int_{0}^{\infty}\left(l_{i}-t_{i}\right) f_{L_{i}}\left(l_{i}\right) d l_{i} \\
+\left[\frac{z}{k}+D t_{m}+\frac{z\left(\sum_{j=1}^{m} k^{j-1}+(n-m) k^{m-1}\right)}{2}\left(1-\frac{1}{k}\right)-\frac{z\left\{\sum_{j=1}^{m} k^{2(j-1)}+(n-m) k^{2(m-1)}\right\}}{2\left\{\sum_{j=1}^{m} k^{j-1}+(n-m) k^{m-1}\right\}}\right] h
\end{gathered}
$$

\subsubsection{Constraints}

Note that the manufacturer needs a notification time $S_{t}+z / P+t_{i}$ from buyer $i$ before finishing the last batch of the current lot meeting demand at this buyer, to maintain its mean lead time $t_{i}$. In this situation if $r_{i}$ is the notification point from the buyer $i$ to the manufacturer, then

$$
z / P+t_{i}+S_{t}=r_{i} / D_{i} \Rightarrow r_{i}=D_{i}\left(S_{t}+z / P+t_{i}\right)
$$

So, if $r$ is the general notification point to the manufacturer to start processing of a next lot to maintain its mean lead time $t_{i}$ considering all buyers, $r$ is given by expression (10):

$$
r=\operatorname{Max}_{i}\left\{D_{i}\left(S_{t}+z / P+t_{i}\right)\right\}
$$

Also, in order to maintain the reorder point, $z_{i} \geq r_{i} \Rightarrow z \geq D r_{i} / D_{i}\left[\because z / D=z_{i} / D_{i}\right]$ and hence

$$
z \geq D\left(S_{t}+t_{i}\right) /(1-1 / k)
$$

In addition, the time of meeting demand by a batch $z_{i}$ cannot be less than sum of the transfer time $t_{i}$ and return time $t_{i}^{\prime}$ of the transport vehicle, which implies,

$$
z_{i} / D_{i} \geq t_{i}+t_{i}^{\prime} . \Rightarrow z \geq D\left(t_{i}+t_{i}^{\prime}\right)\left[\because z / D=z_{i} / D_{i}\right]
$$

Besides, the largest batch $k^{m-1} z_{i}$ cannot exceed the capacity $g$ of the transport vehicle, which implies, 


$$
k^{m-1} z_{i} \leq g \Rightarrow k^{m-1} z D_{i} / D \leq g \Rightarrow z \leq D g /\left(k^{m-1} D_{i}\right)
$$

Moreover, $z_{i} \geq 1 \Rightarrow z D_{i} / D \geq 1 \Rightarrow z \geq D / D_{i}$. So, considering expressions (11), (12) and (13), we obtain

$$
\underline{z}=\operatorname{Maximum}(D 1, D 2, D 3) \leq z \leq \bar{z} \text { Or } \underline{z} \leq z \leq \bar{z}
$$

where $D 1=\operatorname{Max}\left\{D\left(S_{t}+t_{i}\right) /(1-1 / k)\right\}, D 2=\operatorname{Max}\left\{D\left(t_{i}+t_{i}^{\prime}\right\}, D 3=\operatorname{Max}\left\{D / D_{i}\right\}, \bar{z}=D g \operatorname{Min}\left\{1 / D_{i}\right\} / k^{m-1}\right.$

Therefore, the objective of the model for the single-manufacturer multi-buyer integrated supply chain problem with generic distribution of lead times is to find the minimum of the total cost function as given in expression (8) by satisfying the constraint of expression (14). Given $z$, the notification point $r$ can be calculated from expression (10).

\section{Solution Algorithm}

Given $n$ and $m$, convexity of the total cost function $T C(z, n, m)$ in $z$ (assuming $z$ as real) is elucidated in Appendix A, while convexity of this total cost function in $n$, with given $z, m$ (assuming $n$ as real), is indicated in Appendix B. Given $z, n$ and $m$, evaluations of the integrals in the total cost function using eight probability distribution functions are illustrated in Appendix C.

From (14) we have $\underline{z} \leq z \leq \bar{z}=D g /\left\{k^{m-1} \operatorname{Min}\left(1 / D_{i}\right)\right\}$. Note that for given parameter values, $\underline{z}$ is fixed.

However, $\bar{z}$ decreases as the value of $m$ increases. So, determine the value of $m$, say $m_{0}$, so that

$$
D g /\left\{k^{m_{0}+1} \operatorname{Min}\left(1 / D_{i}\right)\right\}<\bar{z} \leq D g /\left\{k^{m_{0}} \operatorname{Min}\left(1 / D_{i}\right)\right\} .
$$

Then starting with $m=1$ and $z=\underline{z}$, find the minimal $n$ by a binary search over $n$ and hence, the associated minimal total cost. For the known values of $m$ and $n$, find the minimal $z$ by a binary search over $z$ satisfying constraint (14) and the associated minimal total cost. Again, for the known minimal values of $m$ and $z$, find the minimal $n$ and the associated minimal total cost, and hence, for the known minimal values of $n$ and $m$, find the minimal $z$ satisfying constraint (14) and the associated minimal total cost. Continue with this process of calculating the minimal $n$ and $z$ for known $m$ along with the associated 
minimal total cost until there is no significant difference between two consecutive minimal total costs. Record the final minimal total cost along with the associated values of $z, n$ and $m$. Increase the current value of $m$ by 1 at each step and find the minimal total cost along with the associated values of $z, n$ and $m$ successively following the above procedure until $m$ exceeds $m_{0}$. Thus, minimum of all the minimal total costs found along with the associated values of $z, n$ and $m$ is the minimal total cost solution to the problem. The solution algorithm is presented below.

\section{Algorithm}

Step 1: Set $m=1$ and $z=\underline{z}$. Increase $m$ by 1 successively until

$$
D g /\left\{k^{m+1} \operatorname{Min}\left(1 / D_{i}\right)\right\}<\bar{z} \leq D g /\left\{k^{m} \operatorname{Min}\left(1 / D_{i}\right)\right\} . \text { Set } m_{0}=m \& m=1 \text {. }
$$

Step 2: For the known values of $m$ and $z$, find the minimal total cost by increasing or decreasing $n$ by 1 at each step but keeping $n \geq m$. Record this minimal total cost as $C^{\prime}$ and set $C_{m}=C^{\prime}$.

Step 3: Keeping fixed the current values of $n$ and $m$, find the minimal total cost by a binary search over $z$ satisfying constraint (14). Record this minimal total cost as $C^{\prime \prime}$. If $C^{\prime \prime}<C_{m}$, set $C_{m}=C^{\prime \prime}, z=$ the minimal $z$ and go to step 2 .

Step 4: Record $C_{m}$ along with the associated values of $z, n$ and $m$.

Step 5: Set $m=m+1$. If $m<m_{0}+1$, go to step 2 .

Step 6: Find the minimal total cost as minimum of $\left\{C_{1}, C_{2}, \ldots C_{m_{0}}\right\}$ with associated values of $n, m$ and $z$. Stop.

\section{Computational Results and Analyses}

\subsection{Solution to numerical problems with various distributions of lead times}

A single-manufacturer 5-buyer numerical problem of a car exhaust silencer manufacturer producing 3200 silencers per year and selling these products to five different car producing plants with a total annual rate 
of demand of 1000 silencers is considered in this numerical example. Table 1 illustrates the relevant data for this case, using most of the deterministic data from Hoque (2008), where integrated manufacturerbuyers inventory models with deterministic lead time were developed. However, the car manufacturers' (i.e. buyers) data for $\pi_{i}, t_{i}, t_{i}^{\prime}$ and $\sigma_{i}$ emphasise on deviations within a certain range.

Table 1: Data set 1 for a single manufacturer 5-buyer numerical problem

\begin{tabular}{|c|c|c|c|c|c|c|c|c|c|c|c|c|}
\hline$P$ & $S$ & $h$ & $g$ & $S_{t}$ & $D_{i}$ & $S_{i}$ & $\boldsymbol{h}_{i}$ & $\pi_{i}$ & $t_{i}$ & $t_{i}$, & $T_{i}$ & $\sigma_{i}$ \\
\hline \multirow[t]{5}{*}{3200} & 400 & 4 & 300 & 0.001 & 200 & 25 & 7 & 30 & 0.009 & 0.003 & 25 & 0.002 \\
\hline & & & & & 180 & 15 & 6 & 27 & 0.007 & 0.003 & 20 & 0.001 \\
\hline & & & & & 225 & 25 & 7.5 & 31 & 0.008 & 0.004 & 18 & 0.003 \\
\hline & & & & & 230 & 30 & 6.5 & 25 & 0.007 & 0.002 & 25 & 0.001 \\
\hline & & & & & 165 & 30 & 6 & 28 & 0.006 & 0.0025 & 15 & 0.0015 \\
\hline
\end{tabular}

$D=\sum_{i=1}^{b} D_{i}=1000$

This numerical problem is solved considering eight different continuous probability distributions of lead time following the algorithm developed. Note that when $m=1$, a lot is delivered with equal batch sizes; when $n=m$ all batches of a lot are unequal sizes; when $m<n$, a lot is delivered by combining equal and unequal batch sizes. Comparative results with $m=1, n=m$ and $m<n$ are given in Table 2.

Table 2: Comparative results obtained for 8 continuous probabilitry distributions of lead times with $m=1, n=m \& m<n$

\begin{tabular}{|c|c|c|c|c|c|c|c|c|c|c|c|c|c|c|c|c|c|}
\hline \multirow[b]{2}{*}{ Distribution } & \multicolumn{5}{|c|}{ Solution with $\mathbf{m}=1$} & \multicolumn{5}{|c|}{ Solution with $m=n$} & \multirow{2}{*}{$\begin{array}{c}\% \\
\text { reduction } \\
\text { in MTC } \\
\text { with } m=n \\
\text { over } m=1\end{array}$} & \multicolumn{5}{|c|}{ Solution with $m<n$} & \multirow{2}{*}{$\begin{array}{c}\% \\
\text { reduction } \\
\text { in MTC } \\
\text { with } \\
m<n \\
\text { over } \mathbf{m}=\mathbf{n}\end{array}$} \\
\hline & $z$ & $n$ & $\begin{array}{c}Q= \\
n z\end{array}$ & $r$ & $\begin{array}{c}\text { Min } T \\
\text { Cost } \\
(M T C)\end{array}$ & $Z$ & $m$ & $Q$ & $r$ & $\begin{array}{c}\text { Min } T \\
\text { Cost }\end{array}$ & & $z$ & $n, m$ & $Q$ & $r$ & $\begin{array}{c}\operatorname{Min} T \\
\operatorname{Cos} t\end{array}$ & \\
\hline Normal & 204 & 3 & 612 & 16.50 & 2765.94 & 39 & 3 & 563.16 & 4.77 & 2728.17 & 1.37 & 86 & 3,2 & 636.40 & 8.07 & 2660.25 & 2.49 \\
\hline Uniform & 122 & 5 & 610 & 10.61 & 3410.02 & 86 & 2 & 361.20 & 8.07 & 4054.70 & -18.91 & 44 & 5,2 & 607.20 & 5.19 & 3396.50 & 16.23 \\
\hline Gamma & 204 & 3 & 612 & 16.50 & 2765.99 & 127 & 2 & 533.40 & 11.43 & 2749.54 & 0.60 & 86 & 3,2 & 636.40 & 8.07 & 2660.30 & 3.25 \\
\hline Exponential & 204 & 3 & 512 & 16.50 & 2769.40 & 39 & 3 & 563.16 & 4.77 & 2750.36 & 0.69 & 86 & 3,2 & 636.40 & 8.07 & 2663.58 & 3.16 \\
\hline Chi-square & 206 & 3 & 618 & 16.65 & 2981.88 & 39 & 3 & 563.16 & 4.77 & 2943.36 & 1.29 & 87 & 3,2 & 643.80 & 8.14 & 2867.17 & 3.59 \\
\hline Beta & 204 & 3 & 612 & 16.50 & 2764.01 & 127 & 2 & 533.40 & 11.43 & 2747.56 & 0.60 & 86 & 3,2 & 636.40 & 8.07 & 2658.32 & 3.25 \\
\hline Weibull & 204 & 3 & 612 & 16.50 & 2763.97 & 127 & 2 & 533.40 & 11.43 & 2747.52 & 0.60 & 86 & 3,2 & 636.40 & 8.07 & 2658.28 & 3.25 \\
\hline Rayleigh & 204 & 3 & 612 & 16.50 & 2762.34 & 127 & 2 & 533.40 & 11.43 & 2745.74 & 0.60 & 86 & 3,2 & 636.40 & 8.07 & 2656.63 & 3.25 \\
\hline
\end{tabular}

It is observed that the percentages of total cost reductions by transferring the lot with $m<n$ instead of $m=$ 1 and $n=m$ are considerable except for Uniform distribution with $n=m$ over $m=1$. To see further the effect of transferring a lot with a combination of equal and/or unequal batch sizes on the minimal total cost, we solve a numerical problem for the same exhaust silencer manufacturer producing 4000 silencers per year and selling these products to five different car producing plants with a set of different data given in Table 3. Comparative results are given in Table 4. 
Table 3: Data set 2 for a single manufacturer 5-buyer numerical problem

\begin{tabular}{ccccccccccccc}
\hline $\boldsymbol{P}$ & $\boldsymbol{S}$ & $\boldsymbol{h}$ & $\boldsymbol{g}$ & \multirow{2}{*}{$\boldsymbol{S}_{\boldsymbol{t}}$} & $\boldsymbol{D}_{\boldsymbol{i}}$ & $\boldsymbol{S}_{\boldsymbol{i}}$ & $\boldsymbol{h}_{\boldsymbol{i}}$ & $\boldsymbol{\pi}_{\boldsymbol{i}}$ & $\boldsymbol{t}_{\boldsymbol{i}}$ & $\boldsymbol{t}_{\boldsymbol{i}}$ & $\boldsymbol{T}_{\boldsymbol{i}}$ & $\boldsymbol{\sigma}_{\boldsymbol{i}}$ \\
\hline 4000 & 400 & 5 & 300 & 0.001 & 400 & 30 & 8 & 27 & 0.009 & 0.003 & 30 & 0.002 \\
\hline & & & 150 & 18 & 6 & 30 & 0.007 & 0.003 & 25 & 0.001 \\
\hline & & & 350 & 30 & 8 & 35 & 0.009 & 0.004 & 18 & 0.003 \\
\hline & & & 190 & 40 & 7 & 25 & 0.007 & 0.002 & 30 & 0.001 \\
\hline
\end{tabular}

$D=\sum_{i=1}^{b} D_{i}=1500$

Table 4: Comparative results obtained for 8 continuous probability distributions of lead times with $m=1, n=m \& m<n$

\begin{tabular}{|c|c|c|c|c|c|c|c|c|c|c|c|c|c|c|c|c|c|}
\hline \multirow[b]{2}{*}{ Distribution } & \multicolumn{5}{|c|}{ Solution with $m=1$} & \multicolumn{5}{|c|}{ Solution with $m=n$} & \multirow{2}{*}{$\begin{array}{c}\% \\
\text { reduction } \\
\text { in MTC } \\
\text { with } m=n \\
\text { over } m=1\end{array}$} & \multicolumn{5}{|c|}{ Solution with $m<n$} & \multirow{2}{*}{$\begin{array}{c}\% \\
\text { reduction in } \\
\text { MTC with } \\
m<n \text { over } \\
m=n\end{array}$} \\
\hline & $z$ & $n$ & $\begin{array}{c}Q= \\
n z\end{array}$ & $r$ & $\begin{array}{c}\text { Min } T \\
\text { Cost } \\
(M T C)\end{array}$ & $z$ & $M$ & $Q$ & $r$ & $\begin{array}{c}\text { Min } T \\
\text { Cost }\end{array}$ & & $z$ & $n, m$ & $Q$ & $r$ & $\begin{array}{c}\text { Min } T \\
\text { Cost }\end{array}$ & \\
\hline Normal & 247 & 3 & 750 & 29.01 & 3865.06 & 57 & 3 & 615.54 & 9.70 & 3720.27 & 3.75 & 124 & 3,2 & 786.16 & 16.40 & 3654.37 & 1.77 \\
\hline Uniform & 150 & 5 & 750 & 19.07 & 4742.50 & 48 & 3 & 518.35 & 8.80 & 5401.89 & -13.90 & 65 & 5,2 & 759.2 & 10.50 & 4673.08 & 13.49 \\
\hline Gamma & 247 & 3 & 741 & 29.01 & 3865.13 & 57 & 3 & 615.54 & 9.70 & 3773.80 & 2.36 & 124 & 3,2 & 786.16 & 16.40 & 3654.43 & 3.16 \\
\hline Exponential & 247 & 3 & 741 & 29.01 & 3873.26 & 57 & 3 & 615.54 & 9.70 & 3769.28 & 2.68 & 124 & 3,2 & 786.16 & 16.40 & 3662.06 & 2.84 \\
\hline Chi-square & 250 & 3 & 750 & 29.32 & 4249.95 & 57 & 3 & 615.54 & 9.70 & 4150.12 & 2.35 & 125 & 3,2 & 792.50 & 16.50 & 4031.15 & 2.87 \\
\hline Beta & 247 & 3 & 741 & 29.01 & 3862.35 & 57 & 3 & 615.54 & 9.70 & 3771.03 & 2.36 & 124 & 3,2 & 786.16 & 16.40 & 3651.39 & 3.17 \\
\hline Weibull & 247 & 3 & 741 & 29.01 & 3860.09 & 57 & 3 & 615.54 & 9.70 & 3770.71 & 2.32 & 124 & 3,2 & 786.16 & 16.40 & 3651.39 & 3.16 \\
\hline Rayleigh & 247 & 3 & 741 & 29.01 & 3860.51 & 57 & 3 & 615.54 & 9.70 & 3769.49 & 2.36 & 124 & 3,2 & 786.16 & 16.40 & 3649.73 & 3.18 \\
\hline
\end{tabular}

Note that the percentages of total cost reductions for this problem increase significantly in all cases except for Uniform as the case for the previous problem (Table 1). This achievement is the result of better synchronisation of the production flow by transferring lots with a combination of equal and/or unequal batch sizes. For both the problems the solutions with Normal distribution of lead times have the smallest minimal total cost whereas the solutions with Uniform and Chi-square distributions of lead times have the highest and the second highest minimal total costs respectively. The presented numerical outcomes (Table 4) are significantly different for Uniform, Chi-square and the set of all other distributions. For the first problem the solutions with Normal, Gamma, Exponential, Beta, Weibull and Rayleigh distributions of lead times are found to provide almost the same result. For the second problem the same trend follows except for Normal distribution. It can be noted that in certain cases the Weibull distribution is very similar to the Normal distribution, and Rayleigh distribution is a particular case of Weibull distribution. The same results follow with these distributions of lead time might be due to their similarities. Additionally, Gamma and Exponential distributions of lead times result in almost the same solution. Since Exponential distribution is a particular case of Gamma distribution, these solutions may be due to their similarities. 
Although this trend of results cannot be concluded generally with the solutions to two numerical problems only, it certainly hints for better results with such synchronisation. Also, the solution method developed here can certainly ensure an optimal solution to a single-manufacturer multi-buyer case with any continuous probability distribution of lead times.

\subsection{Comparative study on single-manufacturer single-buyer numerical problems}

Since similar treatment of the problem does not exist in the literature, like-to-like comparison on solutions to numerical problems cannot be conducted. However, we perform comparative studies on solutions to single-manufacturer single-buyer numerical problem using the proposed method, and some earlier methods with normal distribution of lead times. In this case we consider the same exhaust silencer manufacturer producing 3000 silencers per year and selling these products to one car producing plant with annual rate of demand of 1000. The data for this problem is adopted from Hoque (2013a) (Table 5) and comparative results are provided in Table 6.

Table 5: Data for a single manufacturer single-buyer numerical problem

\begin{tabular}{ccccccccccccc}
\hline $\boldsymbol{P}$ & $\boldsymbol{S}$ & $\boldsymbol{h}$ & $\boldsymbol{g}$ & $\boldsymbol{S}_{\boldsymbol{t}}$ & $\boldsymbol{D}_{\boldsymbol{i}}$ & $\boldsymbol{S}_{\boldsymbol{i}}$ & $\boldsymbol{h}_{\boldsymbol{i}}$ & $\pi_{i}$ & $\boldsymbol{t}_{\boldsymbol{i}}(\mathbf{y r})$ & $\boldsymbol{t}_{\boldsymbol{i}}{ }^{\prime}(\mathbf{y r})$ & $\boldsymbol{T}_{\boldsymbol{i}}$ & $\sigma_{\boldsymbol{i}}$ \\
\hline 3000 & 400 & 4 & 300 & 0.001 & 1000 & 25 & 5 & 30 & 0.009 & 0 & 0 & 0.002 \\
\hline
\end{tabular}

Table 6: Comparative minimal total cost solutions to three numerical problems with different production rate

\begin{tabular}{|c|c|c|c|c|c|c|}
\hline$P$ & Method & $Z$ & $n,(n, m)$ & $Q$ & $r_{m}$ & $T C$ \\
\hline \multirow[t]{3}{*}{3000} & Hoque (2013a) & 112 & 5 & 560 & 47.33 & 1983.72 \\
\hline & Hoque (2013b) & 15 & $(4,4)$ & 600 & 15 & 1635.38 \\
\hline & This Paper & 15 & $(13,2)$ & 915 & 12.60 & 1584.68 \\
\hline \multirow[t]{3}{*}{5000} & Hoque (2013a) & 127 & 4 & 508 & 35.4 & 2037.11 \\
\hline & Hoque (2013b) & 17 & $(3,3)$ & 527 & 13.40 & 1739.84 \\
\hline & This Paper & 27 & $(19,1)$ & 513 & 15.4 & 1721.66 \\
\hline \multirow[t]{3}{*}{7000} & Hoque (2013a) & 158 & 3 & 474 & 32.57 & 2043.12 \\
\hline & Hoque (2013b) & 33 & $(3,2)$ & 495 & 14.71 & 1735.55 \\
\hline & This Paper & 27 & $(18,1)$ & 486 & 13.86 & 1773.85 \\
\hline
\end{tabular}

For the studied three numerical problems, the proposed method provides lesser minimal total cost solutions in all cases except for $P=7000$ where Hoque (2013b) has provided better solution. Note that the assumed mean lead time in developing the model in this paper is different from that of Hoque (2013a, 2013b). Also, the production flow of this model has been synchronised with the creation of a notification point for starting processing of a next lot. Thus, the variation in the minimal total cost solutions in Table 6 
is due to these differences. However, from Table 6 it can be observed that as the production rate increases from demand the minimal total cost solution is obtained by transferring the lot with equal-sized batches.

\subsection{Sensitivity analyses of the solutions}

To demonstrate the effect of change in the transportation cost on the minimal total cost, we successively increase each of the transportation costs of the single-manufacturer five-buyer numerical problem (data of which is given in Table 3) by 5 units. As the transportation cost increases the minimal batch sizes in each of the distributions are found to be in the increasing order keeping the minimal values of $n$ and $m$ the same. Comparative variations in the minimal total costs are illustrated in Figure 2.

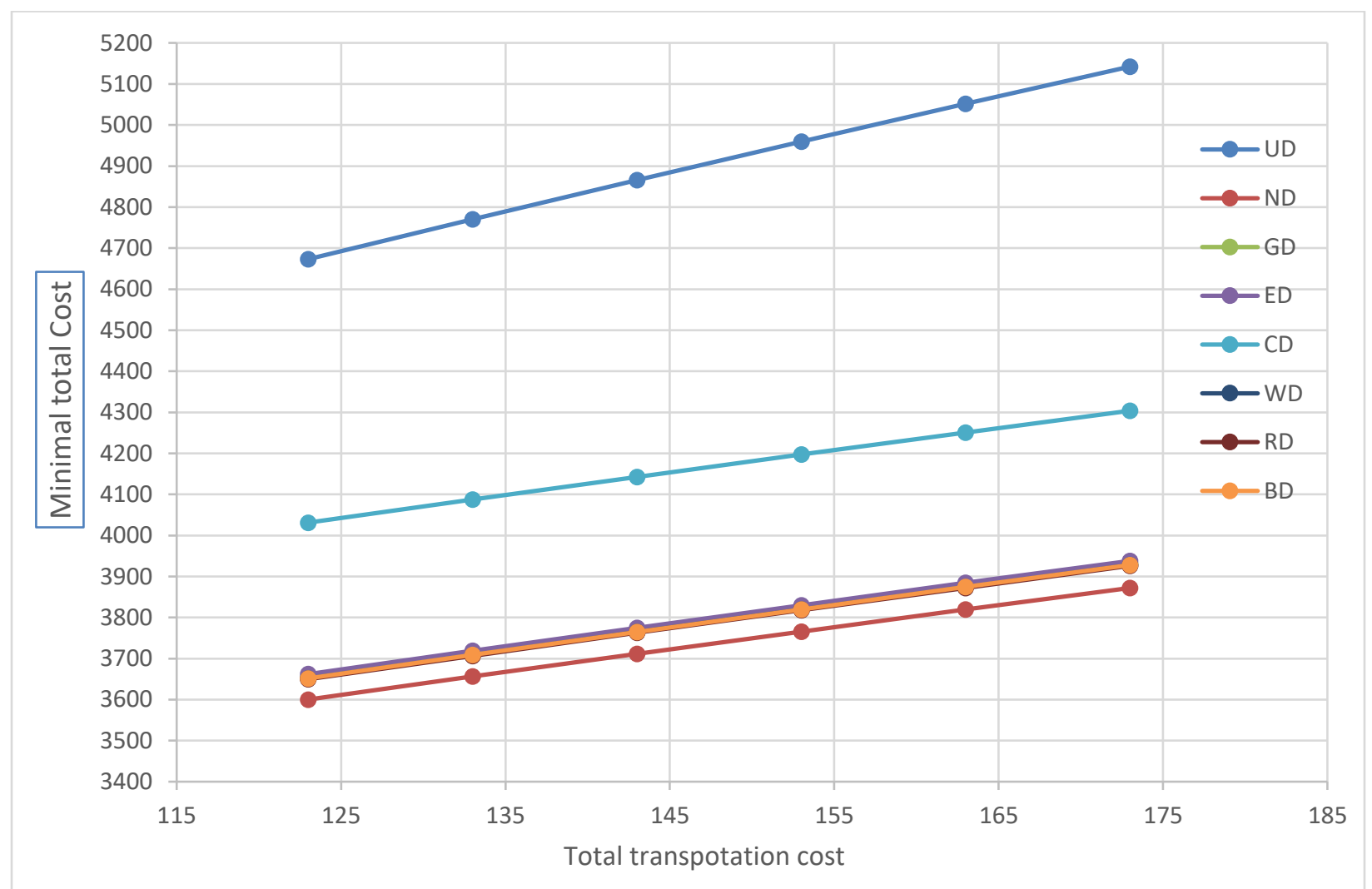

Figure 2: Trends in the minimal total costs in various distribution of lead times for increasing values of the transportation cost $\left(T_{i}\right)$

It is noticed from Figure 2 that as the transport cost increases, the minimal total cost with uniform distribution of lead times has the highest growth rate. The minimal total costs with all other distributions 
have the similar smaller growth rates. The minimal total system cost is found to increase by $10.03 \%$ in case of uniform distribution whereas the minimal total system costs in other cases are found to increase by around $7.55 \%$.

Similar analysis has been carried out by increasing each of the shortage costs by 5 units in various distributions of lead times. The minimal batch sizes and the associated values of $n$ and $m$ in each of the distributions are found to be the same. As the shortage cost increases the variation in the minimal total cost for different distributions of lead times is illustrated in Figure 3.

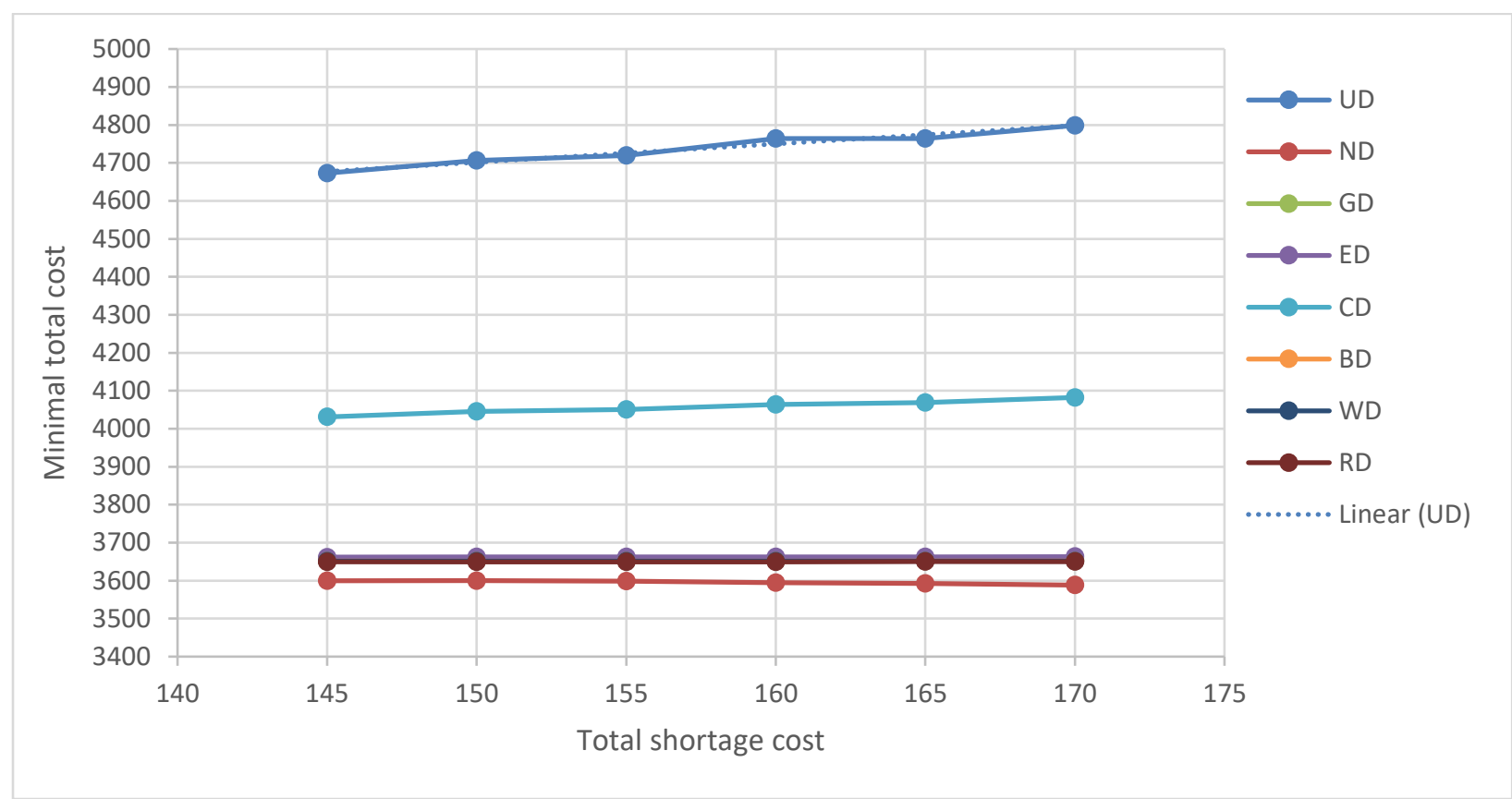

Figure 3: Trends in the minimal total costs in various distribution of lead times for increasing values of the shortage cost $\left(\pi_{i}\right)$

Figure 3 illustrates that the minimal total cost has the highest growth rate in case of uniform distribution and the next highest growth rate in it with Chi-square distribution. In the former case, the minimal total system cost is found to increase by $2.69 \%$ whereas it increases in the latter case by $1.27 \%$. In all other cases almost similar insignificant growth rates were found.

The minimal total cost solutions for successive increases in each of the inventory costs by 1 unit for various distributions of lead times are also calculated. As the inventory cost increases, the minimal batch 
sizes are found in decreasing trend except for Uniform where they are the same, and the associated values of $n$ and $m$ are observed to be the same. The corresponding variation in the minimal total costs in various distributions of lead times is illustrated in Figure 4. In all cases the minimal total cost increases. It has the smallest growth rate in case of Uniform distribution with $0.66 \%$ increase in the minimal total system cost, and the highest in case of Normal distribution with $5.44 \%$ increase in the same. In all other cases the minimal total system cost increases by around $3.95 \%$.

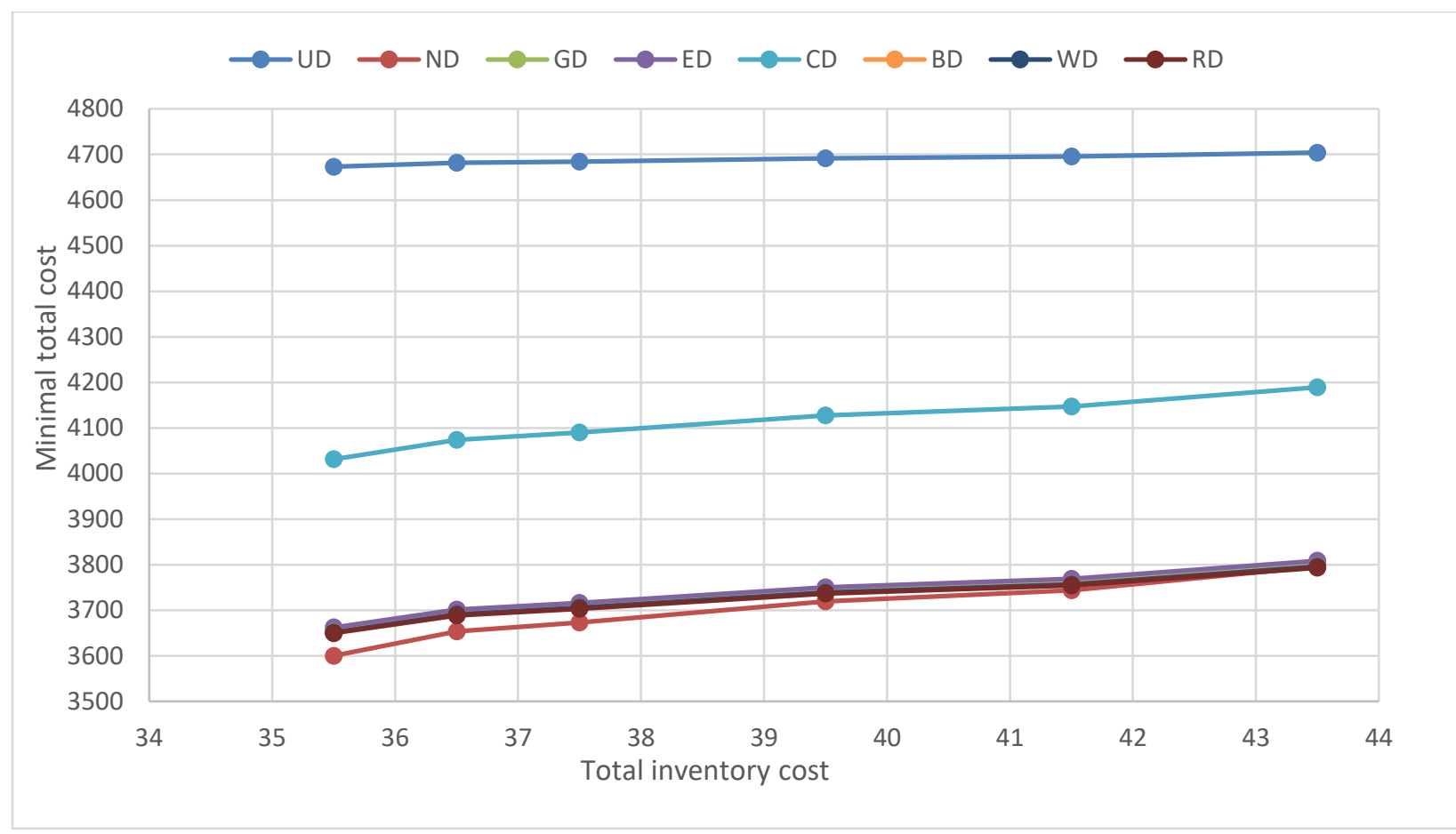

Figure 4: Trends in the minimal total costs in various distribution of lead times for increasing values of $h_{\mathrm{i}}$

The minimal total cost solutions for successive equal increases in $t_{i}$ by 0.002 units for different distributions of lead times are computed and depicted in Figure 5. As the value of $t_{i}$ increases, the minimal lot sizes and the associated values of $n$ and $m$ in each of the distributions are found to be the same. As $t_{i}$ increases, initially the minimal total cost increases $(0.25 \%$ with uniform distribution, $0.94 \%$ with Chi-square and around $0.40 \%$ with others) and then it remains almost the same in all cases except for Chi-square, where it has the highest growth rate along with $2.58 \%$ increase in the minimal total system 
cost. Note that the initial increase in the minimal total cost is due to the increase in $D h t_{m}$ (the annual inventory cost during transportation), which is remained unchanged because of no change in $t_{m}$ during successive increases in other $t_{i}$ latter.

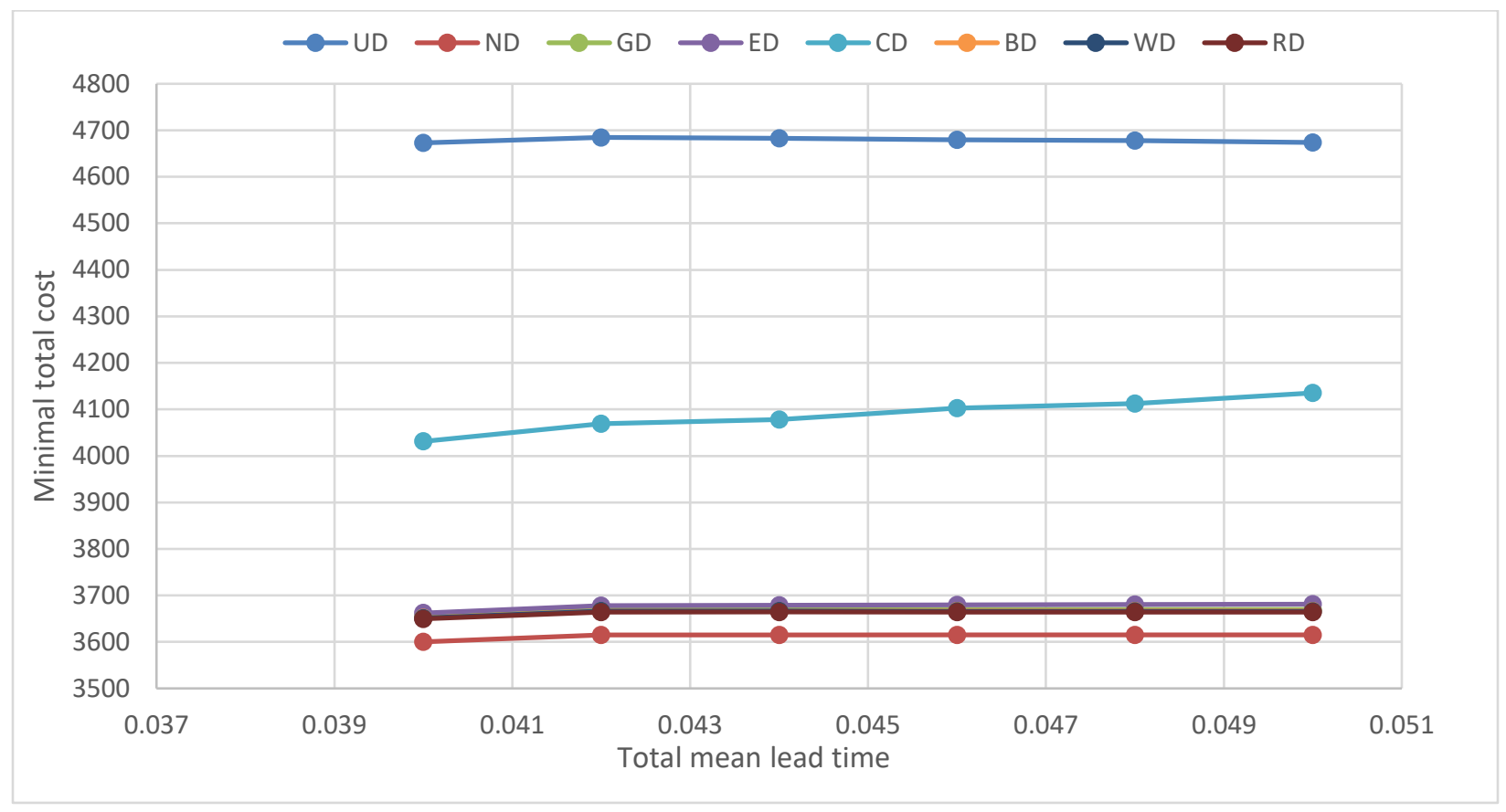

Figure 5: Trends in the minimal total costs in various distribution of lead times for increasing values of $t_{i}$

\subsection{Managerial implications}

Variable lead times often lead to a higher degree of uncertainty. The harmful impact of this variability on production and distribution systems is widely accepted by practitioners and academics (Heydari et al., 2016). de Treville et al. (2014) reported that managers struggle to quantify the actual financial impact of lead times - which greatly obstructs their decision making in this system. However, in practice, lead times of delivering batches of a product to a buyer may be approximated to a kind of continuous probability distribution. Since the model in this paper has been developed with generic continuous probability distribution of lead times, a manager can easily implement this model along with such a distribution. Generally, a production manager deals with delivering of their produced products to multiple buyers. As the literature is silent on a single-manufacturer multi-buyer integrated production inventory model with 
stochastic lead times of delivering batches of a lot, a manager has the opportunity to assess the financial impact of variable lead time in such a situation. The production flow of the integrated model in this paper has been synchronised by delivering the lot with equal and/or unequal batch sizes, and with the establishment of a notification point to the buyer for starting production of a next lot. This has opened a new scope to managers for finding minimal total cost solution to a problem with such synchronisation and employing stochastic lead times of delivering equal and/or unequal batch sizes of a lot. Thus the technique developed in this paper has augmented the scope of applications of a required technique to a further extent for the managers.

\section{Conclusions}

This article develops an integrated single-manufacturer multi-buyer model with generic continuous probability distribution of lead times of delivering equal and/or unequal batch sizes of a lot. The production-distribution supply chain flow is synchronised by synchronising the transfer of batches and generating a notification point to start processing of a next lot in time. The smallest batch size delivered to a buyer is constrained to be greater than or equal to the notification point from the corresponding buyer, demand of this buyer at the transfer plus return time of a transport vehicle and 1, and it is also kept less than or equal to the capacity of the transport vehicle. The total cost of ordering, set up, inventory holding, shortage and transportation is minimised by applying the method of differentiation, and it is illustrated with numerical example problems. Potential benefit of the model is highlighted with solutions of numerical example problems. Sensitivity analyses performed by increasing the values of transportation and inventory costs, illustrate significant increase in the minimal total system cost except for uniform in the latter. For increasing values of the shortage cost and the mean lead time the minimal total system cost increases considerably in case of Chi-square, whereas it increases notably with uniform in the former. Besides, variation in the mean lead times is found to have insignificant effect on the minimal total system cost, which is in line with the conclusion made based on throughput-based analysis by Ponte et al. (2018). 
Demands of the buyers are assumed to be a deterministic constant. However, in practice, the demands of buyers may vary under the influence of various factors. Further research on this topic can be conducted considering this variation. Generally, distribution of the profit among the concerned parties based on negotiation is suggested by researchers. Nonetheless, researchers can focus on finding a reasonable way to distribute the earned profit (from the integrated system) among the concerned parties.

\section{Appendix A}

Proof of convexity of the total cost function with respect to the smallest batch size, $z$ keeping $n, m$ constant

Given $n$ and $m$, assuming $z$ as real, differentiate the total cost function $T(z, n, m)$ with respect to $z$ (Leibnitz theorem of differentiation of an integral is used to differentiate an integral) to obtain

$$
\begin{aligned}
& \frac{d T(z, n, m)}{d z}=\sum_{i=1}^{b}\left[\frac{D_{i} h_{i}}{2 D}\left\{\frac{\sum_{j=1}^{m} k^{2(j-1)}+(n-m) k^{2(m-1)}}{\sum_{j=1}^{m} k^{j-1}+(n-m) k^{m-1}}\right\} \int_{0}^{t_{i}} f_{L_{i}}\left(l_{i}\right) d l_{i}\right] \\
& -\frac{1}{\left\{\sum_{j=1}^{m} k^{j-1}+(n-m) k^{m-1}\right\}} \sum_{i=1}^{b} D_{i}\left(\pi_{i}+h_{i}\right)\left[\sum_{j=1}^{m} k^{2(j-1)} \int_{t_{i}}^{t_{i}+k^{j-1} z / D}\left\{\frac{\left(l_{i}-t_{i}\right)}{z}-\frac{k^{j-1}}{D}\right\} f_{L_{i}}\left(l_{i}\right) d l_{i}\right. \\
& \left.+\frac{D}{2 z^{2}} \sum_{j=1}^{m} k^{j-1}\left\{\int_{t_{i}}^{t_{i}+k^{j-1} z / D}\left(\left(l_{i}-t_{i}\right)-\frac{z k^{j-1}}{D}\right)^{2} f_{L_{i}}\left(l_{i}\right) d l_{i}\right\}\right] \\
& -\frac{(n-m)}{\left\{\sum_{j=1}^{m} k^{j-1}+(n-m) k^{m-1}\right\}} \sum_{i=1}^{b} D_{i}\left(\pi_{i}+h_{i}\right)\left[k^{m-1} \int_{t_{i}}^{t_{i}+k^{m-1} z / D}\left\{\frac{\left(l_{i}-t_{i}\right)}{z}-\frac{k^{m-1}}{D}\right\} f_{L_{i}}\left(l_{i}\right) d l_{i}\right. \\
& \left.+\frac{D}{2 z^{2}}\left\{\int_{t_{i}}^{t_{i}+k^{m-1} z / D}\left(\left(l_{i}-t_{i}\right)-\frac{z k^{m-1}}{D}\right)^{2} f_{L_{i}}\left(l_{i}\right) d l_{i}\right\}\right] \\
& -\frac{1}{\sum_{j=1}^{m} k^{j-1}+(n-m) k^{m-1}} \sum_{i=1}^{b} D_{i} \pi_{i}\left[\sum_{j=1}^{m}\left(\frac{k^{j-1}}{2 D}\right) \int_{t_{i}}^{\infty} f_{L_{i}}\left(l_{i}\right) d l_{i}+(n-m) \frac{k^{m-1}}{2 D} \int_{t_{i}}^{\infty} f_{L_{i}}\left(l_{i}\right) d l_{i}\right] \\
& +\left[\frac{1}{k}+\frac{\left(\sum_{j=1}^{n} k^{j-1}+(n-m) k^{m-1}\right)}{2}\left(1-\frac{1}{k}\right)-\frac{\left\{\sum_{j=1}^{m} k^{2(j-1)}+(n-m) k^{2(m-1)}\right\}}{2\left\{\sum_{j=1}^{m} k^{j-1}+(n-m) k^{m-1}\right\}}\right] h-\frac{D\left[S+\sum_{i=1}^{b}\left(S_{i}+n T_{i}\right)\right]}{z^{2}\left\{\sum_{j=1}^{m} k^{j-1}+(n-m) k^{m-1}\right\}}
\end{aligned}
$$




$$
\begin{aligned}
& \Rightarrow \frac{d^{2} T(z, n, m)}{d z^{2}}=\frac{1}{\left\{\sum_{j=1}^{m} k^{j-1}+(n-m) k^{m-1}\right\}} \sum_{i=1}^{b} D_{i}\left(\pi_{i}+h_{i}\right)\left[\sum_{j=1}^{m} k^{2(j-1)} \int_{t_{i}}^{t_{i}+k^{j-z} z / D}\left(\frac{l_{i}-t_{i}}{z^{2}}\right) f_{L_{i}}\left(l_{i}\right) d l_{i}\right. \\
& \left.+\frac{1}{z^{2}} \sum_{j=1}^{m} k^{2(j-1)}\left\{\int_{t_{i}}^{t_{i}+j^{-1-z / D}}\left(\left(l_{i}-t_{i}\right)-\frac{z k^{j-1}}{D}\right) f_{L_{i}}\left(l_{i}\right) d l_{i}\right\}+\frac{D}{z^{3}} \sum_{j=1}^{m} k^{j-1}\left\{\int_{t_{i}}^{t_{i}+k^{j-1} z / D}\left(\left(l_{i}-t_{i}\right)-\frac{z k^{j-1}}{D}\right)^{2} f_{L_{i}}\left(l_{i}\right) d l_{i}\right\}\right] \\
& +\frac{(n-m)}{\left\{\sum_{j=1}^{m} k^{j-1}+(n-m) k^{m-1}\right\}} \sum_{i=1}^{b} D_{i}\left(\pi_{i}+h_{i}\right)\left[k^{m-1} \int_{t_{i}}^{t_{i}+k^{n-1} z / D} \frac{\left(l_{i}-t_{i}\right)}{z^{2}} f_{L_{i}}\left(l_{i}\right) d l_{i}+\frac{2 D\left[S+\sum_{i=1}^{b}\left(S_{i}+n T_{i}\right)\right]}{z^{3}\left\{\sum_{j=1}^{m} k^{j-1}+(n-m) k^{m-1}\right\}}\right. \\
& \left.\quad \frac{k^{m-1}}{z^{2}}\left\{\int_{t_{i}}^{t_{i}+k^{m-1} z / D}\left(\left(l_{i}-t_{i}\right)-\frac{z k^{m-1}}{D}\right) f_{L_{i}}\left(l_{i}\right) d l_{i}\right\}+\frac{D}{z^{3}}\left\{\int_{t_{i}}^{t_{i}+k^{m-1} z / D}\left(\left(l_{i}-t_{i}\right)-\frac{z k^{m-1}}{D}\right)^{2} f_{L_{i}}\left(l_{i}\right) d l_{i}\right\}\right] \\
& \quad \frac{d^{2} T(z, n, m)}{d z^{2}}=\frac{1}{\left\{\sum_{j=1}^{m} k^{j-1}+(n-m) k^{m-1}\right\}} \sum_{i=1}^{b} D_{i}\left(\pi_{i}+h_{i}\right)\left[\frac{D}{z^{3}} \sum_{j=1}^{m} k^{j-1}\left\{\int_{t_{i}}^{t_{i}+k^{j-z} z / D}\left(l_{i}-t_{i}\right)^{2} f_{L_{i}}\left(l_{i}\right) d l_{i}\right\}\right] \\
& +\frac{(n-m)}{\left\{\sum_{j=1}^{m} k^{j-1}+(n-m) k^{m-1}\right\}} \sum_{i=1}^{b} D_{i}\left(\pi_{i}+h_{i}\right)\left[\frac{D}{z^{3}}\left(\int_{t_{i}}^{t_{i}+k^{m-1} z / D}\left(l_{i}-t_{i}\right)^{2} f_{L_{i}}\left(l_{i}\right) d l_{i}\right\}\right]+\frac{2 D\left[S+\sum_{i=1}^{b}\left(S_{i}+n T_{i}\right)\right]}{z^{3}\left\{\sum_{j=1}^{m} k^{j-1}+(n-m) k^{m-1}\right\}},
\end{aligned}
$$

which is positive. Hence the function $T(z, n, m)$ is convex in $z$, given $n$ and $m$.

\section{Appendix B}

Proof of convexity of the total cost function with respect to the total number of batches, $n$ keeping $z$,

\section{$m$ constant}

Given $z$ and $m$, differentiate $T(z, n, m)$ with respect to $n$ (assuming $n$ as real) to obtain

$$
\begin{aligned}
& \frac{D T(z, n, m)}{d n}=\frac{\left\{\frac{z\left(k^{m}-1\right)\left(k^{m-1}-1\right) k^{m-1}}{2 D\left(k^{2}-1\right)}\right\}\left\{\sum_{i=1}^{b} D_{i} h_{i} \int_{0}^{t_{i}} f_{L_{i}}\left(l_{i}\right) d l_{i}\right\}+\frac{D}{2 z} \sum_{j=1}^{m} k^{j-1} \sum_{i=1}^{b}\left[D_{i}\left(h_{i}+\pi_{i}\right)\left\{\int_{t_{i}}^{t_{i}+k^{m-1} z / D}\left(\left(l_{i}-t_{i}\right)-\frac{z k^{m-1}}{D}\right)^{2} f_{L_{i}}\left(l_{i}\right) d l_{i}\right\}\right]}{\left\{\sum_{j=1}^{m} k^{j-1}+(n-m) k^{m-1}\right\}^{2}} \\
& +\frac{\sum_{j=1}^{m} k^{j-1} \sum_{i=1}^{b} D_{i}\left[\pi_{i} \int_{t_{i}}^{\infty}\left\{\left(l_{i}-t_{i}-\frac{k^{m-1} z}{2 D}\right)\right\} f_{L_{i}}\left(l_{i}\right) d l_{i}-\frac{D \sum_{i=1}^{b} T_{i}}{z}\left(m k^{m}-\sum_{=1}^{m} k^{j-1}\right)\right]}{\left\{\sum_{j=1}^{m} k^{j-1}+(n-m) k^{m-1}\right\}^{2}} \\
& +\frac{z k^{m-1} h}{2}\left(1-\frac{1}{k}\right)-\frac{1}{\left\{\sum_{j=1}^{m} k^{j-1}+(n-m) k^{m-1}\right\}^{2}}\left[\frac{D\left(S+\sum_{i=1}^{b} S_{i}\right) k^{m-1}}{z}+\frac{z h\left(k^{m}-1\right)\left(k^{m-1}-1\right) k^{m-1}}{2\left(k^{2}-1\right)}\right. \\
& \left.+\frac{D k^{m-1}}{2 z} \sum_{i=1}^{b} D_{i}\left(\pi_{i}+h_{i}\right)\left\{\sum_{j=1}^{m} \int_{t_{i}}^{t_{i}+k^{j-1} z / D}\left(\left(l_{i}-t_{i}\right)-\frac{z k^{j-1}}{D}\right)^{2} f_{L_{i}}\left(l_{i}\right) d l_{i}\right\}+k^{m-1} \sum_{i=1}^{b} D_{i} \pi_{i}\left\{\sum_{j=1}^{m} \int_{t_{i}}^{\infty}\left(l_{i}-t_{i}-\frac{k^{j-1} z}{2 D}\right) f_{L_{i}}\left(l_{i}\right) d l_{i}\right\}\right]
\end{aligned}
$$




$$
\begin{aligned}
& \Rightarrow \frac{d^{2}(z, n, m)}{d n^{2}}=\frac{z\left(k^{m}-1\right)\left(k^{m-1}-1\right) k^{2(m-1)}}{\left(k^{2}-1\right)\left\{\sum_{j=1}^{m} k^{j-1}+(n-m) k^{m-1}\right\}^{3}}\left[h-\frac{1}{D} \sum_{i=1}^{b} D_{i} h_{i} \int_{0}^{t_{i}} f_{L_{i}}\left(l_{i}\right) d l_{i}\right] \\
& +\frac{2 k^{m-1}\left[k^{m-1} \sum_{i=1}^{b} D_{i} \pi_{i}\left\{\sum_{j=1}^{m} \int_{t_{i}}^{\infty}\left(l_{i}-t_{i}-\frac{k^{j-1} z}{2 D}\right) f_{L_{i}}\left(l_{i}\right) d l_{i}\right\}-\sum_{j=1}^{m} k^{j-1} \sum_{i=1}^{b} D_{i} \pi_{i} \int_{t_{i}}^{\infty}\left\{\left(l_{i}-t_{i}-\frac{k^{m-1} z}{2 D}\right)\right\} f_{L_{i}}\left(l_{i}\right) d l_{i}\right]}{\left\{\sum_{j=1}^{m} k^{j-1}+(n-m) k^{m-1}\right\}^{3}} \\
& +\frac{D k^{m-1} \sum_{i=1}^{b} D_{i}\left(\pi_{i}+h_{i}\right)\left[k^{m-1}\left\{\sum_{j=1}^{m} \int_{t_{i}}^{t_{i}+k^{j-z} z / D}\left(\left(l_{i}-t_{i}\right)-\frac{z k^{j-1}}{D}\right)^{2} f_{L_{i}}\left(l_{i}\right) d l_{i}\right\}-\sum_{j=1}^{m} k^{j-1}\left\{\int_{t_{i}}^{t_{i}+k^{m-1} z / D}\left(\left(l_{i}-t_{i}\right)-\frac{z k^{m-1}}{D}\right)^{2} f_{L_{i}}\left(l_{i}\right) d l_{i}\right\}\right]}{z\left\{\sum_{j=1}^{m} k^{j-1}+(n-m) k^{m-1}\right\}^{3}} \\
& +\frac{2 D k^{m-1}\left[\left(m k^{m}-\sum_{=1}^{m} k^{j-1}\right) \sum_{i=1}^{b} T_{i}+\left(S+\sum_{i=1}^{b} S_{i}\right) k^{(m-1)}\right]}{z\left\{\sum_{j=1}^{m} k^{j-1}+(n-m) k^{m-1}\right\}^{3}} \\
& \Rightarrow \frac{d^{2}(z, n, m)}{d n^{2}}=\frac{z\left(k^{m}-1\right)\left(k^{m-1}-1\right) k^{2(m-1)}}{\left(k^{2}-1\right)\left\{\sum_{j=1}^{m} k^{j-1}+(n-m) k^{m-1}\right\}^{3}}\left[h-\frac{1}{D} \sum_{i=1}^{b} D_{i} h_{i} \int_{0}^{t_{i}} f_{L_{i}}\left(l_{i}\right) d l_{i}\right] \\
& +\frac{2 k^{m-1}\left[k^{m-1} \sum_{i=1}^{b} D_{i} \pi_{i}\left\{\sum_{j=1}^{m} \int_{t_{i}}^{\infty}\left(l_{i}-t_{i}\right) f_{L_{i}}\left(l_{i}\right) d l_{i}\right\}-\sum_{j=1}^{m} k^{j-1} \sum_{i=1}^{b} D_{i} \pi_{i} \int_{t_{i}}^{\infty}\left\{\left(l_{i}-t_{i}\right)\right\} f_{L_{i}}\left(l_{i}\right) d l_{i}\right]}{\left\{\sum_{j=1}^{m} k^{j-1}+(n-m) k^{m-1}\right\}^{3}} \\
& +\frac{D k^{m-1} \sum_{i=1}^{b} D_{i}\left(\pi_{i}+h_{i}\right)\left[\left\{k^{m-1} \sum_{j=1}^{m} \int_{t_{i}}^{t_{i}+k^{-1} z / D}\left(\left(l_{i}-t_{i}\right)-\frac{z k^{j-1}}{D}\right)^{2} f_{L_{i}}\left(l_{i}\right) d l_{i}\right\}-\sum_{j=1}^{m} k^{j-1}\left\{\int_{t_{i}}^{t_{i}+k^{m-1} z / D}\left(\left(l_{i}-t_{i}\right)-\frac{z k^{m-1}}{D}\right)^{2} f_{L_{i}}\left(l_{i}\right) d l_{i}\right\}\right]}{z\left\{\sum_{j=1}^{m} k^{j-1}+(n-m) k^{m-1}\right\}^{3}} \\
& +\frac{2 D k^{m-1}\left[\left(m k^{m}-\sum_{=1}^{m} k^{j-1}\right) \sum_{i=1}^{b} T_{i}+\left(S+\sum_{i=1}^{b} S_{i}\right) k^{(m-1)}\right]}{z\left\{\sum_{j=1}^{m} k^{j-1}+(n-m) k^{m-1}\right\}^{3}} \\
& \frac{D k^{m-1}}{z} \sum_{i=1}^{b} D_{i}\left(\pi_{i}+h_{i}\right) k^{m-1}\left\{\sum_{j=1}^{m} \int_{t_{i}}^{t_{i}+k^{j-1} z / D}\left(\left(l_{i}-t_{i}\right)-\frac{z k^{j-1}}{D}\right)^{2} f_{L_{i}}\left(l_{i}\right) d l_{i}\right\}-\sum_{j=1}^{m} k^{j-1}\left\{\int_{t_{i}}^{t_{i}+k^{m-1} z / D}\left(\left(l_{i}-t_{i}\right)-\frac{z k^{m-1}}{D}\right)^{2} f_{L_{i}}\left(l_{i}\right) d l_{i}\right\} \\
& =\frac{D k^{m-1}}{z} \sum_{i=1}^{b} D_{i} \pi_{i} k^{m-1}\left\{\sum_{j=1}^{m} \int_{t_{i}}^{t_{i}+k^{j-1} z / D}\left(\left(l_{i}-t_{i}\right)-\frac{z k^{j-1}}{D}\right)^{2} f_{L_{i}}\left(l_{i}\right) d l_{i}\right\}-\sum_{j=1}^{m} k^{j-1}\left\{\int_{t_{i}}^{t_{i}+k^{m-1} z / D}\left(\left(l_{i}-t_{i}\right)-\frac{z k^{m-1}}{D}\right)^{2} f_{L_{i}}\left(l_{i}\right) d l_{i}\right\} \\
& +\frac{D k^{m-1}}{z} \sum_{i=1}^{b} D_{i} h_{i}\left[\sum_{j=1}^{m} k^{j-1}\left\{\int_{0}^{k^{m-j_{i}}+k^{m-1} z / D}\left(\left(l_{i}-t_{i}\right)-\frac{z k^{j-1}}{D}\right)^{2} f_{L_{i}}\left(l_{i}\right) d l_{i}\right\}-\sum_{j=1}^{m} k^{j-1}\left\{\int_{0}^{t_{i}+k^{m-1} z / D}\left(\left(l_{i}-t_{i}\right)-\frac{z k^{m-1}}{D}\right)^{2} f_{L_{i}}\left(l_{i}\right) d l_{i}\right\}\right. \\
& \left.+\sum_{j=1}^{m} k^{j-1}\left\{\int_{0}^{t_{i}}\left(\left(l_{i}-t_{i}\right)-\frac{z k^{m-1}}{D}\right)^{2} f_{L_{i}}\left(l_{i}\right) d l_{i}\right\}-k^{m-1} \sum_{j=1}^{m} \int_{0}^{t_{i}}\left(\left(l_{i}-t_{i}\right)-\frac{z k^{j-1}}{D}\right)^{2} f_{L_{i}}\left(l_{i}\right) d l_{i}\right] \\
& =\frac{D k^{m-1}}{z} \sum_{i=1}^{b} D_{i} \pi_{i} k^{m-1}\left\{\sum_{j=1}^{m} \int_{t_{i}}^{k^{m-j_{i}}+k^{m-1} z / D}\left(\left(l_{i}-t_{i}\right)-\frac{z k^{j-1}}{D}\right)^{2} f_{L_{i}}\left(l_{i}\right) d l_{i}\right\}-\sum_{j=1}^{m} k^{j-1}\left\{\int_{t_{i}}^{t_{i}+k^{m-1} z / D}\left(\left(l_{i}-t_{i}\right)-\frac{z k^{m-1}}{D}\right)^{2} f_{L_{i}}\left(l_{i}\right) d l_{i}\right\}
\end{aligned}
$$




$$
\begin{array}{r}
+\frac{D k^{m-1}}{z} \sum_{i=1}^{b} D_{i} h_{i}\left[\sum_{j=1}^{m} k^{j-1}\left\{\int_{0}^{k^{m-t_{i}+k^{m-1} z / D}}\left(\left(l_{i}-t_{i}\right)-\frac{z k^{j-1}}{D}\right)^{2} f_{L_{i}}\left(l_{i}\right) d l_{i}\right\}-\sum_{j=1}^{m} k^{j-1}\left\{\int_{0}^{t_{i}+k^{m-1} z / D}\left(\left(l_{i}-t_{i}\right)-\frac{z k^{m-1}}{D}\right)^{2} f_{L_{i}}\left(l_{i}\right) d l_{i}\right\}\right. \\
\left.+\sum_{j=1}^{m} k^{j-1}\left\{\int_{0}^{t_{i}}\left(\left(l_{i}-t_{i}\right)-\frac{z k^{m-1}}{D}\right)^{2} f_{L_{i}}\left(l_{i}\right) d l_{i}\right\}-k^{m-1} \sum_{j=1}^{m} \int_{0}^{t_{i}}\left(\left(l_{i}-t_{i}\right)-\frac{z k^{j-1}}{D}\right)^{2} f_{L_{i}}\left(l_{i}\right) d l_{i}\right]
\end{array}
$$

Note that the first and the second differences of the above expression are positive. The third difference can be transformed to

$$
\sum_{i=1}^{b} D_{i} h_{i}\left[\frac{D}{z} k^{m-1}\left\{\frac{k^{m}-1}{k-1}-m k^{m-1}\right\} \int_{0}^{t_{i}}\left(l_{i}-t_{i}\right)^{2} f_{L_{i}}\left(l_{i}\right) d l_{i}+\frac{z\left(k^{m}-1\right)\left(k^{m-1}-1\right) k^{2(m-1)}}{D\left(k^{2}-1\right)} \int_{0}^{t_{i}} f_{L_{i}}\left(l_{i}\right) d l_{i}\right]
$$

Adding it (along with the corresponding denominator in $d^{2}(z, n, m) / d n^{2}$ ) with its first term obtain

$$
\frac{\frac{z h\left(k^{m}-1\right)\left(k^{m-1}-1\right) k^{2(m-1)}}{\left(k^{2}-1\right)}+\sum_{i=1}^{b} D_{i} h_{i}\left[\frac{D}{z} k^{m-1}\left\{\frac{k^{m}-1}{k-1}-m k^{m-1}\right\} \int_{0}^{t_{i}}\left(l_{i}-t_{i}\right)^{2} f_{L_{i}}\left(l_{i}\right) d l_{i i}\right]}{\left\{\sum_{j=1}^{m} k^{j-1}+(n-m) k^{m-1}\right\}^{3}}
$$

The numerator can be transformed to:

$$
\begin{aligned}
& k^{2(m-1)}\left\{\frac{z h\left(k^{m}-1\right)\left(k^{m-1}-1\right)}{\left(k^{2}-1\right)}-m \frac{D}{z} \sum_{i=1}^{b} D_{i} h_{i} \int_{0}^{t_{i}}\left(l_{i}-t_{i}\right)^{2} f_{L_{i}}\left(l_{i}\right) d l_{i}\right\}+\frac{D\left(k^{m}-1\right) k^{m-1}}{z(k-1)} \sum_{i=1}^{b} D_{i} h_{i} \int_{0}^{t_{i}}\left(l_{i}-t_{i}\right)^{2} f_{L_{i}}\left(l_{i}\right) d l_{i} \\
& =m k^{2(m-1)}\left\{\frac{z h\left(k^{m}-1\right)\left(k^{m-1}-1\right)}{m\left(k^{2}-1\right)}-\frac{D}{z} \sum_{i=1}^{b} D_{i} h_{i} \int_{0}^{t_{i}}\left(l_{i}-t_{i}\right)^{2} f_{L_{i}}\left(l_{i}\right) d l_{i}\right\}+\frac{D\left(k^{m}-1\right) k^{m-1}}{m z(k-1)} \sum_{i=1}^{b} D_{i} h_{i} \int_{0}^{t_{i}}\left(l_{i}-t_{i}\right)^{2} f_{L_{i}}\left(l_{i}\right) d l_{i} \quad \text { Let } \\
& \frac{k^{m}-1}{m} \geq \frac{k^{m+1}-1}{m+1} \Rightarrow 0 \geq m k^{m}(k-1)-\left(k^{m}-1\right) \Rightarrow 0 \geq m k^{m}-\frac{k^{m}-1}{k-1}>0[\because k-1>0], \text { a contradiction. Hence }
\end{aligned}
$$

$\left(k^{m}-1\right) / m$ is increasing in $m$. Note that for $m=1$ the above numerator is zero and all other terms in

$d^{2}(z, n, m) / d n^{2}$ are zero except the last one. The last term is positive. For $m>1$, all terms in $\frac{d^{2}(z, n, m)}{d n^{2}}$ are positive and hence the function $T(z, n, m)$ is convex in $n$, given $z$ and $m$. 


\section{Appendix C}

Evaluation of integrals involving various kinds of continuous probability distribution functions

\section{C.1. For the uniform distribution of the lead time:}

Substituting for $f_{L_{i}}\left(l_{i}\right)=\frac{1}{k^{j-1} z+t_{i}-0}=\frac{1}{k^{j-1} z+t_{i}}$ in (8), the integrals there can easily be evaluated.

\section{2. For the normal distribution of the lead time:}

In this case $f_{L_{i}}\left(l_{i}\right)=\frac{1}{\sqrt{2 \pi} \sigma} e^{-\frac{1}{2 \sigma^{2}\left(l_{i}-t_{i}\right)^{2}}}$. We have $\int\left(l_{i}-t_{i}\right) f_{L_{i}}\left(l_{i}\right) d l_{i}=\frac{1}{\sqrt{2 \pi} \sigma_{i}} \int\left(l_{i}-t_{i}\right) e^{-\frac{1}{2 \sigma_{i}^{2}\left(l_{i}-t_{i}\right)^{2}}} d l_{i}$

Substituting for $\left(l_{i}-t_{i}\right)^{2}=u_{i}$ obtains $\int\left(l_{i}-t_{i}\right) f_{L_{i}}\left(l_{i}\right) d l_{i}=-\frac{\sigma_{i}}{\sqrt{2 \pi}} e^{-\frac{\left(l_{i}-t_{i}\right)^{2}}{2 \sigma_{i}^{2}}}$

Also, substituting for $l_{i}-t_{i}=u_{i}$ in $\int\left(l_{i}-t_{i}\right)^{2} f_{L_{i}}\left(l_{i}\right) d l_{i}$ derives

$$
\int\left(l_{i}-t_{i}\right)^{2} f_{L_{i}}\left(l_{i}\right) d l_{i}=-\frac{\sigma_{i}}{\sqrt{2 \pi}}\left(l_{i}-t_{i}\right) e^{-\frac{\left(l_{i}-t_{i}\right)^{2}}{2 \sigma_{i}^{2}}}+\sigma_{i}^{2} \int f_{L_{i}}\left(l_{i}\right) d l_{i}
$$

Using the above integral results, the integrals in (8) can be evaluated.

\section{C.3. For the gamma distribution of the lead time:}

The total cost function (8) in terms of gamma distribution function $f_{L_{i}}\left(l_{i}\right)=\frac{\beta^{-\alpha}}{\Gamma(\alpha)} l_{i}^{\alpha-1} e^{-\frac{l_{i}}{\beta}}, 0 \leq l_{i}<\infty, \alpha>0, \beta>0$ can be assessed by evaluating the integrals $\int l_{i}^{2} f_{L_{i}}\left(l_{i}\right) d l_{i}$ and $\int l_{i} f_{L_{i}}\left(l_{i}\right) d l_{i}$ respectively as follows:

$\alpha(\alpha+1) \beta^{2} \int \frac{\beta^{-(\alpha+2)}}{\Gamma(\alpha+2)} l_{i}^{(\alpha+2)-1} e^{-l_{i} / \beta} d l_{i}$ and $\alpha \beta \int \frac{\beta^{-(\alpha+1)}}{\Gamma(\alpha+1)} l_{i}^{(\alpha+1)-1} e^{-l_{i} / \beta} d l_{i}$

Here the mean lead time, $\alpha \beta=t_{i}$ and the variance $\alpha \beta^{2}=y$ (say), which imply $\alpha=t_{i}^{2} / y, \beta=y / t_{i}$. Thus for given mean lead time $t_{i}$, variance $y$ and other parameter values, the minimal $z, m$ and $n$ can be calculated following the developed algorithm.

\section{C.4. For erlang, exponential and chi-square distributions of the lead time:}


The gamma distribution function transforms to erlang distribution function when $\alpha$ is an integer, and the exponential distribution function is a special case of erlang distribution when $\alpha=1$. Thus the exponential distribution function for the mathematical model in this paper is given by $f_{L_{i}}\left(l_{i}\right)=\frac{1}{t_{i}} e^{-\frac{l_{i}}{t_{i}}}, 0 \leq l_{i}<\infty$, when $\alpha=1$ and $\beta=t_{i}$ are set in the gamma distribution function. So, the minimal $z, m$ and $n$ for each of erlang and exponential distributions can easily be obtained by following the solution procedure described for the case of gamma distribution but by setting $\alpha=1$ and $\beta=t_{i}$.

Chi-square distribution function is a particular case of the gamma distribution function when $\alpha=v / 2$ and $\beta=2$. So, the minimal $z, m$ and $n$ considering chi-square distribution function can be found by setting $\alpha=v / 2, \beta=2$ and $v=t_{i}$ in the gamma distribution function.

\section{C.5. Considering beta distribution of the lead time:}

Consider the total cost function (8) in terms of the beta distribution function

$f_{L_{i}}\left(l_{i}\right)=\frac{1}{B(\alpha, \beta)} l_{i}^{\alpha-1}\left(1-l_{i}\right)^{\beta-1}, 0 \leq l_{i}<\infty, \alpha>0, \beta>0$. The minimal $z, m$ and $n$ can be obtained from (8)

by evaluating the integrals $\int l_{i}^{2} f_{L_{i}}\left(l_{i}\right) d l_{i}$ and $\int l_{i} f_{L_{i}}\left(l_{i}\right) d l_{i}$ respectively as follows:

$\frac{\alpha(\alpha+1)}{(\alpha+\beta)(\alpha+\beta+1)} \int \frac{l_{i}^{(\alpha+2)-1}}{B(\alpha+2, \beta)}\left(1-l_{i}\right)^{\beta-1} d l_{i}$ and $\frac{\alpha}{(\alpha+\beta)} \int \frac{l_{i}^{(\alpha+1)-1}}{B(\alpha+1, \beta)}\left(1-l_{i}\right)^{\beta-1} d l_{i}$, where the mean lead time, $\frac{\alpha}{\alpha+\beta}=t_{i} \quad$ and $\quad$ the $\quad$ variance $\quad \frac{\alpha \beta}{(\alpha+\beta)^{2}(\alpha+\beta+1)}=y \quad$ (say), which imply $\alpha=t_{i}\left\{\left(1-t_{i}\right) t_{i}-y\right\} / y, \beta=\left(1-t_{i}\right)\left(\left\{\left(1-t_{i}\right) t_{i}-y\right\} / y\right.$. Note that $\alpha>0, \beta>0 \Rightarrow 1>t_{i}+y$. Thus for given mean lead time $t_{i}$, variance $y$, and other parameter values, the minimal $z, m$ and $n$ can be calculated following the developed algorithm.

\section{C.6. Considering Weibull distribution of the lead time:}

Consider the mathematical model of the problem in terms of the Weibull distribution function $f_{L_{i}}\left(l_{i}\right)=\alpha \beta^{-\alpha} l_{i}^{\alpha-1} e^{-l_{i}^{\alpha} / \beta^{\alpha}}, 0 \leq l_{i}<\infty, \alpha>0, \beta>0$. 
Following the algorithm the minimal $z, m$ and $n$ can be obtained considering (8) and evaluating the integrals $\int_{t_{i}}^{\infty} l_{i}^{2} f\left(l_{i}\right) d l_{i}, \int_{t_{i}+z / D}^{\infty} l_{i}^{2} f\left(l_{i}\right) d l_{i}, \int_{t_{i}}^{\infty} l_{i} f\left(l_{i}\right) d l_{i}, \int_{t_{i}+z / D}^{\infty} l_{i} f\left(l_{i}\right) d l_{i}$, and $\int_{t_{i}}^{\infty} f_{L_{i}}\left(l_{i}\right) d l_{i}$ as follows:

Assuming $\left(\frac{l_{i}}{\beta}\right)^{\alpha}=\frac{u}{\beta}$, the integrals $\int_{t_{i}}^{\infty} \alpha \beta^{-\alpha} l_{i}^{\alpha-1} e^{-\left(\frac{l_{i}}{\beta}\right)^{\alpha}} l_{i}^{k} d l_{i}$ and $\int_{t_{i}+z l D}^{\infty} \alpha \beta^{-\alpha} l_{i}^{\alpha-1} e^{-\left(\frac{l_{i}}{\beta}\right)^{\alpha}} l_{i}^{k} d l_{i}$ for $k=2,1,0$, transform to the gamma distribution format

$\Gamma(k / \alpha+1) \beta^{k} \int_{\frac{\left(t_{i}\right)^{\alpha}}{\beta^{\alpha-1}}}^{\infty} \frac{\beta^{-(k / \alpha+1)}}{\Gamma(k / \alpha+1)} u^{(k / \alpha+1)-1} e^{-\frac{u}{\beta}} d u$ and $\Gamma(k / \alpha+1) \beta^{k} \int_{\frac{\left(t_{i}+z / D\right)^{\alpha}}{\beta^{\alpha-1}}}^{\infty} \frac{\beta^{-(k / \alpha+1)}}{\Gamma(k / \alpha+1)} u^{(k / \alpha+1)-1} e^{-\frac{u}{\beta}} d u$.

Here the mean lead time, $\beta \Gamma\left(\frac{1}{\alpha}+1\right)=t_{i}$ and the variance $\beta^{2}\left[\Gamma\left(\frac{2}{\alpha}+1\right)-\left\{\Gamma\left(\frac{1}{\alpha}+1\right)\right\}^{2}\right]=y$ (say), which imply

$$
\begin{aligned}
& \left\{\beta \Gamma\left(\frac{1}{\alpha}+1\right)\right\}^{2}\left[\Gamma\left(\frac{2}{\alpha}+1\right) /\left\{\Gamma\left(\frac{1}{\alpha}+1\right)\right\}^{2}-1\right]=y \Rightarrow t_{i}^{2}\left[\Gamma\left(\frac{2}{\alpha}+1\right) /\left\{\Gamma\left(\frac{1}{\alpha}+1\right)\right\}^{2}-1\right]=y\left[\because \beta \Gamma\left(\frac{1}{\alpha}+1\right)=t_{i}\right] \\
& \Rightarrow \Gamma\left(\frac{2}{\alpha}+1\right) /\left\{\Gamma\left(\frac{1}{\alpha}+1\right)\right\}^{2}=\frac{y}{t_{i}^{2}}+1
\end{aligned}
$$

Letting $1 / \alpha=x$, this equation transforms to $\frac{\Gamma(2 x+1)}{\{\Gamma(x+1)\}^{2}}=\frac{y}{t_{i}^{2}}+1$

Now $\frac{\Gamma\{2(x+1)+1\}}{\{\Gamma(x+1)+1\}^{2}}=\frac{2(x+1) \Gamma\{2(x+1\}}{\{(x+1) \Gamma(x+1)\}^{2}}=\frac{2 \Gamma\{(2 x+1)+1\}}{(x+1)\{\Gamma(x+1)\}^{2}}=\frac{2(2 x+1) \Gamma\{(2 x+1)\}}{(x+1)\{\Gamma(x+1)\}^{2}}$

$=2\left(1+\frac{x}{x+1}\right) \frac{\Gamma\{(2 x+1)\}}{\{\Gamma(x+1)\}^{2}}>\frac{\Gamma\{(2 x+1)\}}{\{\Gamma(x+1)\}^{2}}$

This shows that $\Gamma\{(2 x+1)\} /\{\Gamma(x+1)\}^{2}$ is an increasing function of $x$. Thus for the given mean lead time $t_{i}$ and variance $y$, the value of $x$ that satisfies equation (15) can easily be calculated by a binary search over $x$. Using this value of $x$, the value of $\alpha$ and hence the value of $\beta$ can be determined. Using these values of $\alpha$ and $\beta$, the values of $\int_{t_{i}}^{\infty}\left(l_{i}-t_{i}\right)^{2} f\left(l_{i}\right) d l_{i}, \int_{t_{i}+z / D}^{\infty}\left(l_{i}-t_{i}\right)^{2} f\left(l_{i}\right) d l_{i}$, and $\int_{t_{i}}^{\infty} f_{L_{i}}\left(l_{i}\right) d l_{i}$ can be evaluated, and hence the minimal $z, m$ and $n$ considering (8). 
If $\alpha=1$ and the mean lead time $\beta \Gamma(1 / \alpha+1)=t_{i}$, then $\beta=t_{i}$ and the variance $\beta^{2}\left[\Gamma\left(\frac{2}{\alpha}+1\right)-\left\{\Gamma\left(\frac{1}{\alpha}+1\right)\right\}^{2}\right]=\beta^{2}(2-1)=t_{i}^{2}$. So $\quad$ the $\quad$ Weibull distribution function $f_{L_{i}}\left(l_{i}\right)=\alpha \beta^{-\alpha} l_{i}^{\alpha-1} e^{-\frac{l_{i}^{\alpha}}{\beta^{\alpha}}}, 0 \leq l_{i}<\infty, \alpha>0, \beta>0 \quad$ reduces to the exponential distribution function, $\frac{1}{\beta} e^{-\frac{l_{i}}{\beta}}=\frac{1}{t_{i}} e^{-\frac{l_{i}}{t_{i}}}$. A useful subfamily of the Weibull family is determined by letting $\alpha=2$ and $\beta=\sqrt{2} \eta$ as $f_{L_{i}}\left(l_{i}\right)=\frac{1}{\eta^{2}} l_{i} e^{-\frac{1}{2}\left(\frac{l_{i}}{\eta}\right)^{2}}, 0 \leq l_{i}<\infty$, which is the Rayleigh distribution function.

\section{C.7. Considering Rayleigh distribution of the lead time}

Consider the function (8) in terms of the Rayleigh distribution function $f_{L_{i}}\left(l_{i}\right)=\frac{1}{\eta^{2}} l_{i} e^{-\frac{1}{2}\left(\frac{l_{i}}{\eta}\right)^{2}}, 0 \leq l_{i}<\infty$, along with the mean lead time $(\sqrt{\pi / 2}) \eta=t_{i}$ or $\eta=(\sqrt{2 / \pi}) t_{i}$, and the variance $\frac{4-\pi}{2} \eta^{2}=\frac{4-\pi}{2} \cdot \frac{2}{\pi} t_{i}{ }^{2}=\frac{4-\pi}{\pi} t_{i}{ }^{2} . \quad$ Given $\quad$ parameter values, evaluating the integrals $\int_{t_{i}}^{\infty}\left(l_{i}-t_{i}\right)^{2} f\left(l_{i}\right) d l_{i}, \int_{t_{i}+z / D}^{\infty}\left(l_{i}-t_{i}\right)^{2} f\left(l_{i}\right) d l_{i}$ and $\int_{t_{i}}^{\infty} f_{L_{i}}\left(l_{i}\right) d l_{i}$ (as in case of Weibull distribution function, but using $\alpha=2$ and $\beta=\sqrt{2} \eta$ along with $\eta=(\sqrt{2 / \pi}) t_{i}$ ), the minimal $z, m$ and $n$ can be found using (8).

\section{References}

Agrawal, S., Sengupta, N.R., Shanker, K., 2009. Impact of information sharing and lead times on bullwhip effect and on-hand inventory. European Journal of Operational Research 192(2), 576-593.

Arkan, A, Hejazi, S.R., 2012. Coordinating orders in a two echelon supply chain with controllable lead time and ordering cost using the credit period. Computers \& Industrial Engineering 62(1), 56-69.

Barron, Y., Baron, O., 2020. QMCD approach for perishability models: The (S, s) control policy with lead time. IISE Transactions 52(2), 133-150. 
Ben-Daya, M., Hariga, M., 2004. Integrated single vendor single buyer model with stochastic demand and variable lead time. International Journal of production Economics 92(1), 75-80.

Bandaly, D., Satir, A., Shanker, L., 2016. Impact of lead time variability in supply chain risk management. International Journal of Production Economics 180, 88-100.

Chang, H.C., Ouyang, L.Y., Wu, K.S., Ho, C.H., 2006. Integrated vendor-buyer cooperative inventory models with controllable lead time and ordering cost reduction. European Journal of Operational Research 170(2), 481-495.

Chen, F., Drezner, Z., Ryan, J.K., Simchi-Levi, D., 2000. Quantifying the bullwhip effect in a simple supply chain: the impact forecasting, lead times and information. Management Science 46(3), 436443.

Christensen, W.J., Germain, R.N., Birou, L., 2007. Variance vs average: Supply chain lead-time as a predictor of financial performance. Supply Chain Management: An International Journal 12(5), 349357.

Costas, J., Ponte, B., De la Puente, D., Pino, R., Puche, J., 2015. Applying Goldratt's theory of constraints to reduce the bullwhip effect through agent-based modelling. Expert Systems with Applications 42(4), 2049-2060.

Chung, W., Talluri, S., Kovács, G., 2018. Investigating the effects of lead-time uncertainties and safety stocks on logistical performance in a border-crossing JIT supply chain. Computers \& Industrial Engineering 118, 440-450.

de Treville, S., Bicer, I., Chavez-Demoulin, V., Hagspiel, V., Schürhoff, N., Tasserit, C., Wager, S., 2014. Valuing lead times. Journal of Operations Management 32(6), 337-346.

Disney, S.M., Towill, D.R., 2003. The effect of vendor managed inventory (VMI) dynamics on the bullwhip effect in supply chains. International Journal of Production Economics 85(2), 199-215.

Fujiwara, O., Sedarage, D., 1997. An optimal (Q, r) policy for a multipart assembly system under stochastic part procurement lead times. European Journal of Operational Research 100(3), 550-556. 
Glock, C.H., 2009. A comment: "Integrated single-vendor single-buyer model with stochastic demand and variable lead time”. International Journal of Production Economics 122(2), 790-792.

Glock, C.H., 2012. Lead time reduction strategies in a single-vendor-single-buyer integrated inventory model with lot size-dependent lead times and stochastic demand. International Journal of Production Economics 136(1), 37-44.

Gunasekaran, A., Subramanian, N., Papadopoulos, A., 2017. Information technology for competitive advantage within logistics and supply chains: a review, Transportation Research Part E: Logistics and Transportation Review 99, 14-33.

Hayya, J.C., Harrison, T.P., He, X.J., 2011. The impact of stochastic lead time reduction on inventory cost under order crossover. European Journal of Operational Research 211(2), 274-281.

Heydari, J.,Mahmoodi, M., Taleizadeh, A.A., 2016. Lead time aggregation: A three-echelon supply chain model. Transportation Research Part E: Logistics and Transportation Review 89, 215-233.

Hoque, M.A., Goyal, S.K., 2006. A heuristic solution procedure for an integrated inventory system under controllable lead-time with equal and unequal sized batch shipments between a vendor and a buyer. International Journal of production Economics 102(2), 217-225.

Hoque, M.A., 2008. Synchronization in the single-manufacturer multi-buyer integrated inventory supply chain. European Journal of Operational Research 188(3), 811-825.

Hoque, M.A., 2009. An extended vendor-buyer integrated inventory model and its minimal cost solution technique. International Journal of Mathematical Modelling and Numerical Optimisation 1(1/2), 5674.

Hoque, M.A., 2013a. A vendor-buyer integrated production-inventory model with normal distribution of lead time. International Journal of Production Economics 144(2), 409-417.

Hoque, M.A., 2013b. A manufacturer-buyer integrated inventory model with stochastic lead times of delivering equal and/or unequal sized batches of a lot. Computers \& Operations Research 40(11), 2740-2751. 
Hossain, M.S.J, Ohaiba, M.M., Sarker, B.R., 2017. An optimal vendor-buyer cooperative policy under generalized lead-time distribution with penalty cost for delivery lateness. International Journal of Production Economics 188, 50-62.

Hosoda, T., Disney, S.M., Gavirneni, N., 2015. The impact of information sharing, random yield, correlation, and lead times in closed loop supply chain. European Journal of Operational Research $246(3), 827-836$.

Hsiao, Y.C., 2008. A note on integrated single vendor single buyer model with stochastic demand and variable lead time. International Journal of Production Economics 114(1), 294-297.

Humair, S., Ruark, J.D., Tomlin, B., Willems, S.P., 2013. Incorporating stochastic lead times in the guaranteed service model of safety stock optimization. Interfaces 43(5), 421-434.

Isotupa, K.S., Samanta, S.K., 2013. A continuous review (s,Q) inventory system with priority customers and arbitrarily distributed lead times. Mathematical and Computer Modelling 57(5-6), 1259-1269.

Jha, J.K., Shanker, K., 2013. Single-vendor multi-buyer integrated production-inventory model with controllable lead time and service level constraints. Applied Mathematical Modelling 37(4), 17531767.

Kelepouris, T., Miliotis, P., Pramatari, K., 2008. The impact of replenishment parameters and information sharing on the bullwhip effect: a computational study. Computers \& Operations Research 35(11), 3657-3670.

Kollberg, B., Dahlgaard, J.J. Brehmer, P.O., 2006. Measuring lean initiatives in health care services: issues and findings. International Journal of Productivity and Performance management 56(1), 7-24.

Kouvelis, P., Tang, S.Y., 2011. On optimal expediting policy for supply systems with uncertain leadtimes. Production and Operations Management 21(2), 309-330.

Li, Y., Xu, X., Zhao, X., Yeung, J.H.Y., Ye F., 2012. Supply chain coordination with controllable lead time and asymmetric information. European Journal of Operational Research 217(1), 108-119.

Pan, J.C., Hsiao, Y.C., 2005. Integrated inventory models with controllable lead time and backorder discount consideration. International Journal of Systems Science 93-94, 387-397. 
Pan, J.C., Yang, J., 2002. A study of an integrated inventory with controllable lead time. International Journal of production Research 40(5), 1263-1273.

Ponte, B., Costas, J., Puche, J., Pino, R., de la Fuente, D., 2018. The value of lead time reduction and stabilization: A comparison between traditional and collaborative supply chains. Transportation Research Part E: Logistics and Transportation Review 111, 165-185.

Sajadieh, M.S., Jokar, M.R.A., Modarres, M., 2009. Developing a coordinated vendor-buyer model in two-stage supply chains with stochastic lead times. Computers \& Operations Research 36(8), 24842489.

Sajadieh, M.S., Thorstenson, A., 2014. Comparing sourcing strategies in two-echelon supply chains. Computers \& Operations Research 45, 108-115.

Song, J.S., Yano, C.A., Lerssrisyuria, P., 2000. Contract assembly: Dealing with combined supply lead time and demand quantity uncertainty. Manufacturing \& Service Operations Management 2(3), 287296.

Song, J-S, Zhang, H., Hou, Y., Wang, M., 2010. The effect of lead time and demand uncertainties in (r, q) inventory systems. Operations Research 58(1), 68-80.

Taleizadeh, A.A., Niaki, S.T.A., Barzinpour, F., 2011. Multiple-buyer multiple-vendor multi-product multi-constraint supply chain problem with stochastic demand and variable lead-time: A harmony search algorithm. Applied Mathematics and Computation 217(22), 9234 -9253.

Ye, F., Xu, X., 2010. Cost allocation model for optimizing supply chain inventory with controllable lead time. Computers \& industrial Engineering 59(1), 93-99.

Yeung, J.H.Y., Ye, F., 2012. Supply chain coordination with controllable lead time and asymmetric information, European Journal of Operational Research 217, 108-119. 